Supplementary Information

\title{
Microbially Synthesized Polymeric Amyloid Fiber Promotes $\beta$-Nanocrystal Formation and Displays Gigapascal Tensile Strength
}

Authors: Jingyao $\mathrm{Li}^{1}$, Yaguang Zhu ${ }^{1}$, Han $\mathrm{Yu}^{1}$, Bin Dai ${ }^{1}$, Young-Shin Jun ${ }^{1}$, Fuzhong Zhang ${ }^{1-3 *}$

\begin{abstract}
Affiliations:
${ }^{1}$ Department of Energy, Environmental and Chemical Engineering,

${ }^{2}$ Division of Biological \& Biomedical Sciences,

${ }^{3}$ Institute of Materials Science \& Engineering,

Washington University in St. Louis, One Brookings Drive, Saint Louis, MO 63130, USA

*Correspondence to: $\underline{\text { fzhang@seas.wustl.edu }}$
\end{abstract}

Keywords: amyloid, silk, protein fibers, wet spinning, protein engineering, wide-angle x-ray diffraction 

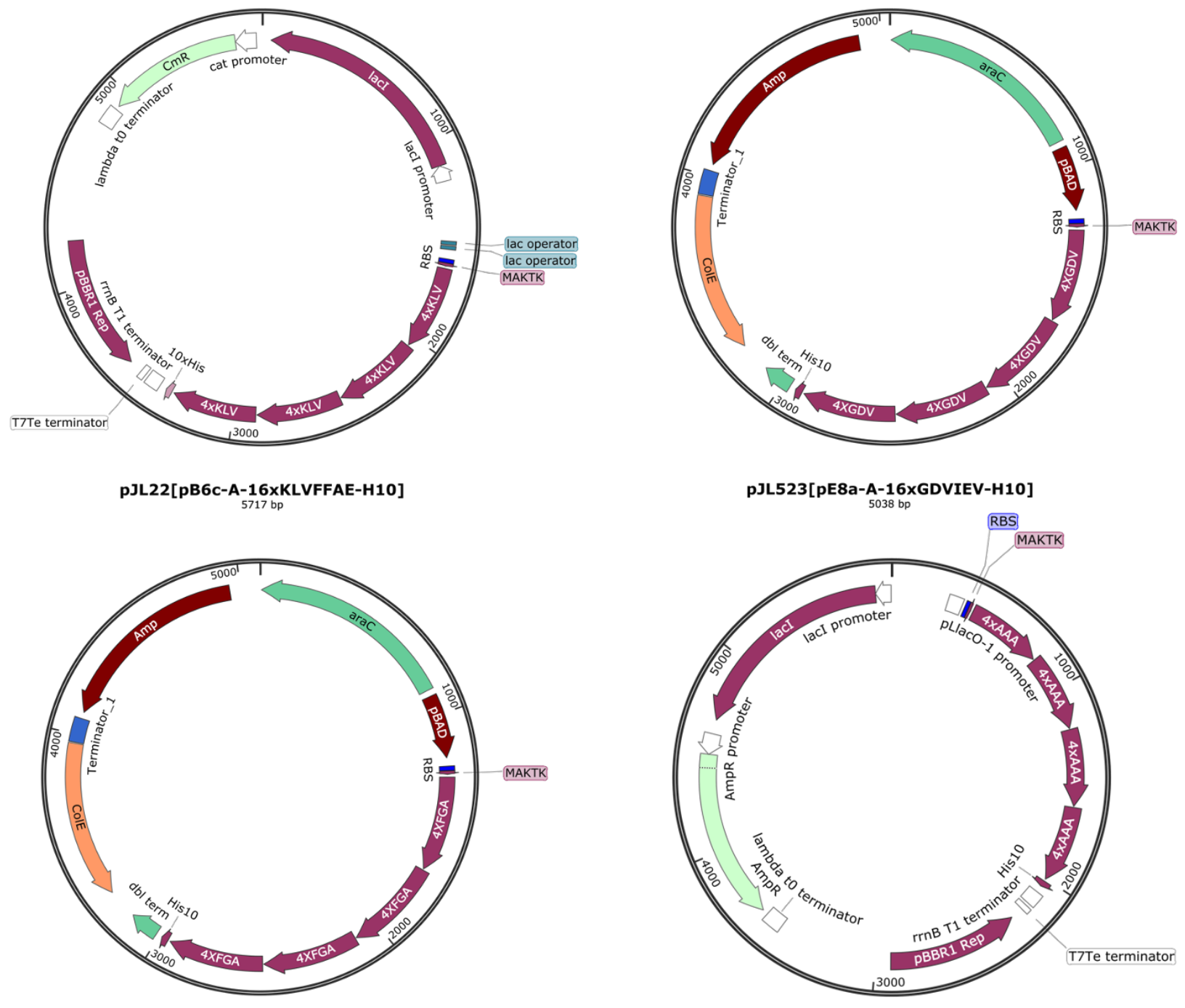

pJL464[pE8a-A-16xFGAILSS-H10]

pJL56[pB6a-A-16xAAAAA-H10]

Fig S1. Plasmid maps used for expression of 16x polymeric amyloid proteins and recombinant silk proteins. 

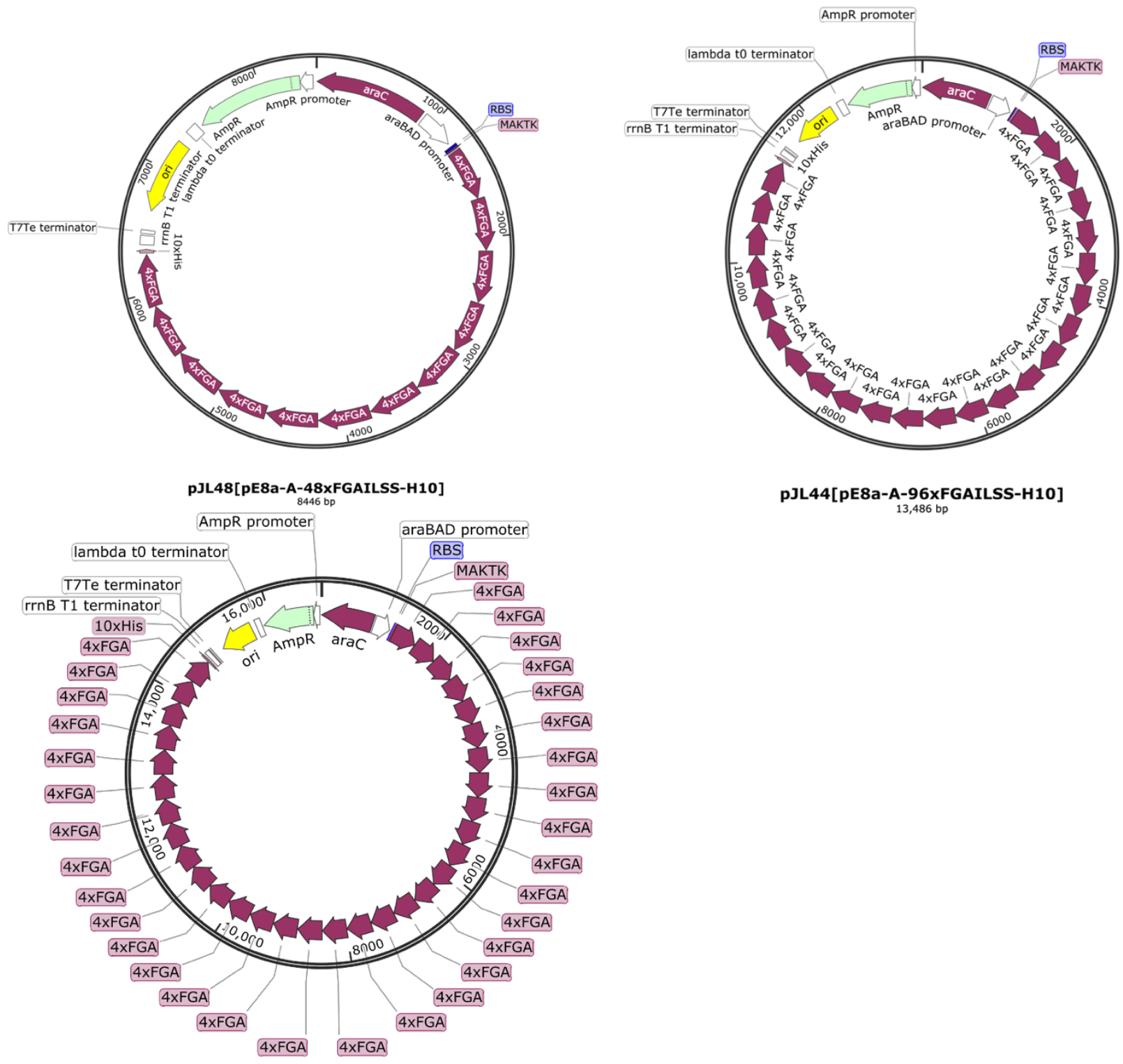

pJL44[pE8a-A-96xFGAILSS-H10] 13,486 bp

pJL51[pE8a-A-128xFGAILSS-H10] 16,846 bp

Fig S2. Plasmids maps used for expressing 48x, 96x, and 128x FGAILSS proteins. 


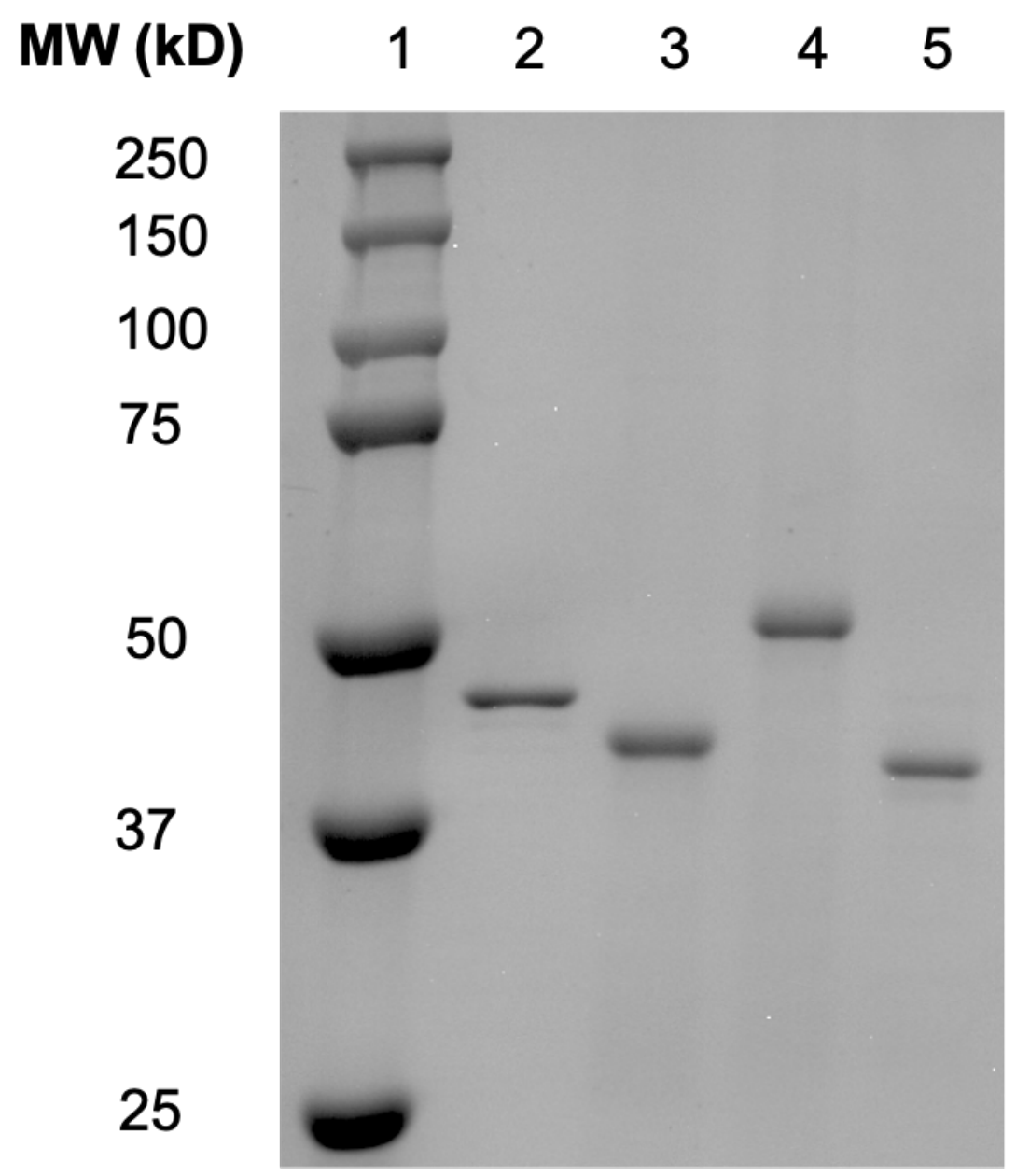

Fig S3. Coomassie blue-stained 10\% SDS-PAGE gel of Ni-NTA affinity chromatography purified 16x polymeric proteins. Lane 1, molecular weight marker with their sized labeled on the left. Lane 2, 16xKLVFFAE protein. Lane 3, 16xFGAILSS protein. Lane 4, 16xGDVIEV protein. Lane 5, 16x recombinant silk protein. 


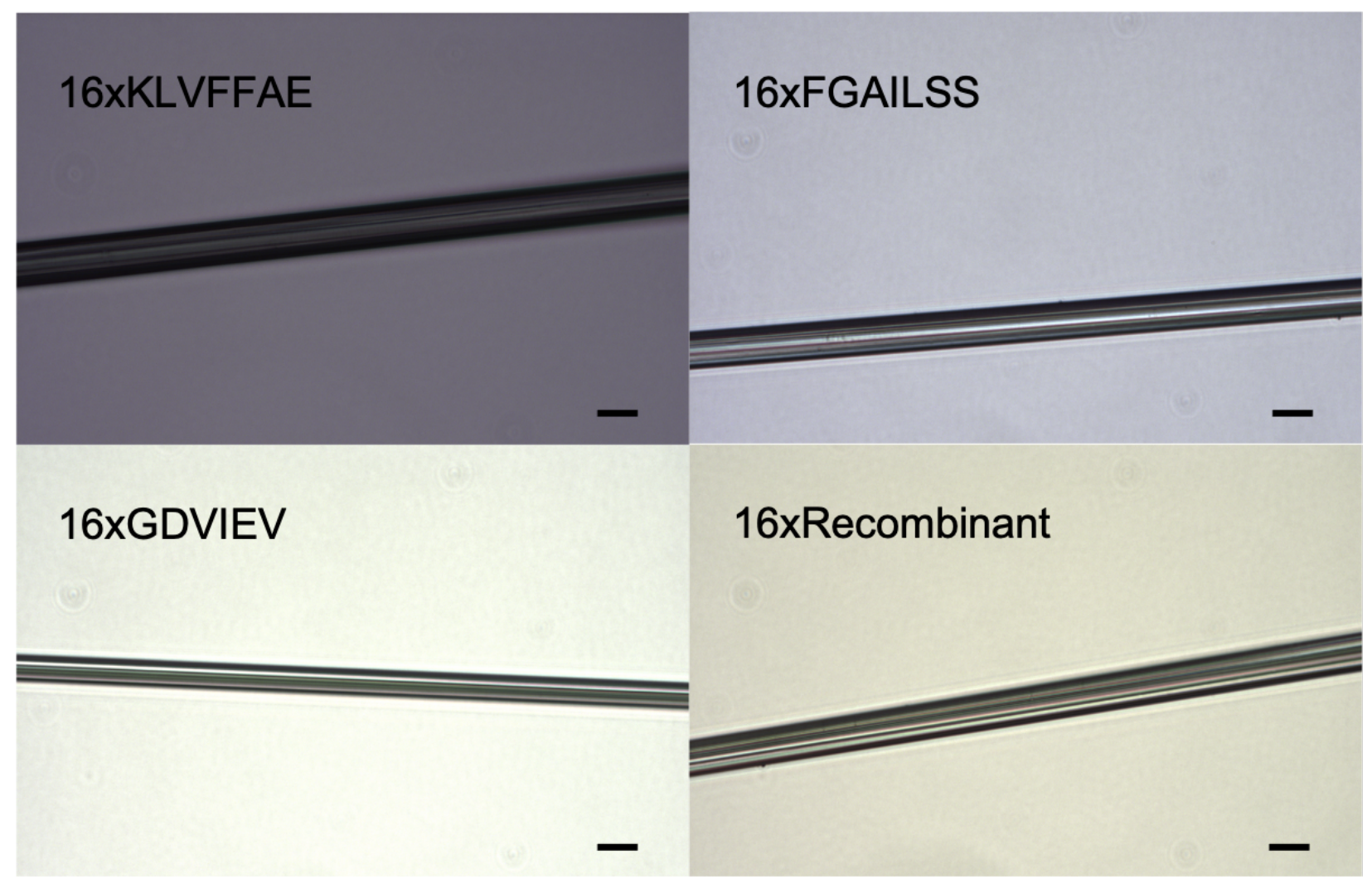

Fig S4. Representative optical images of 16x fibers. Scale bars (black lines) represent $20 \mu \mathrm{m}$. 

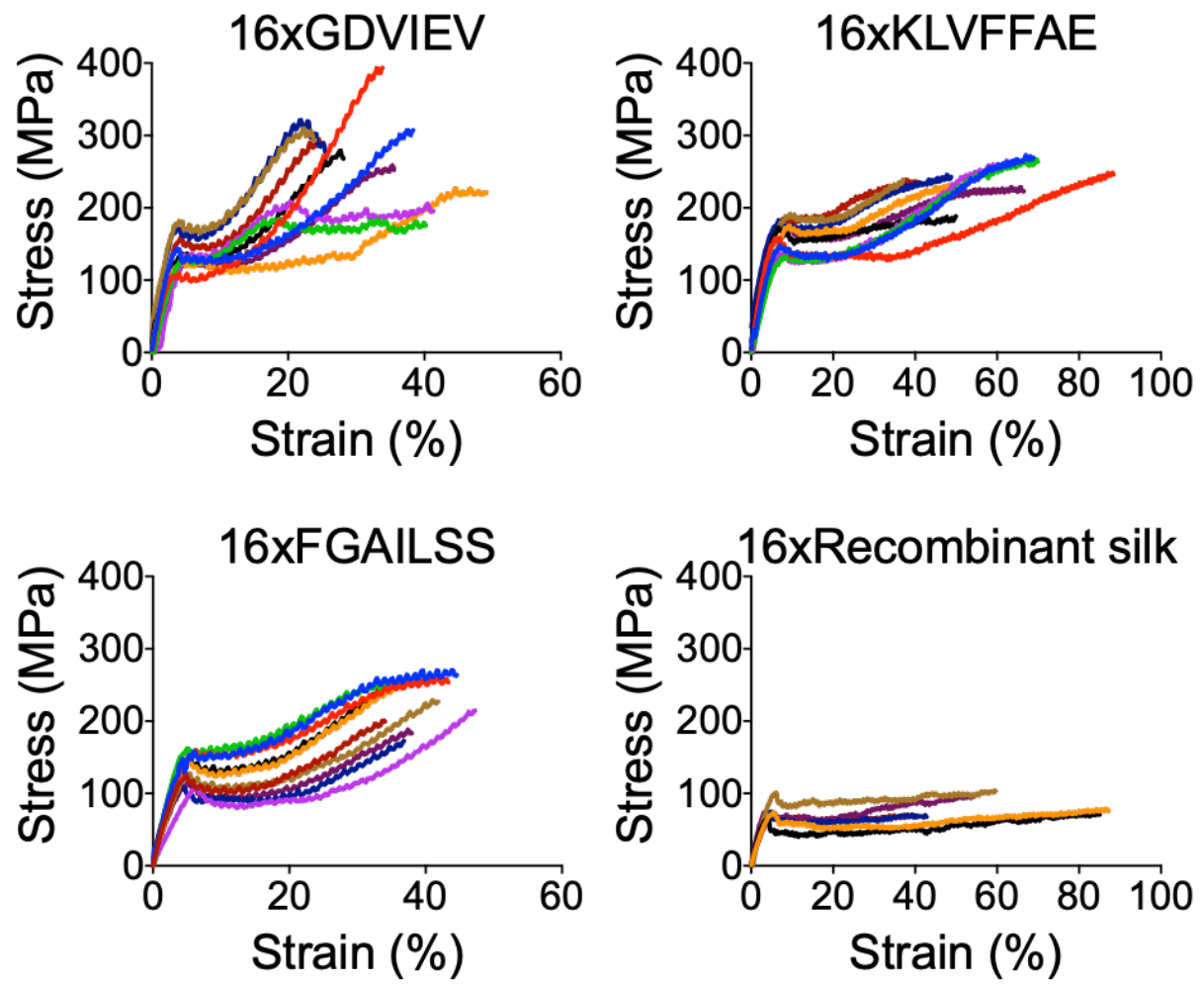

Fig S5. Stress-strain curves of 10 individual 16x polymeric amyloid fibers. Each color represents an individual fiber. 


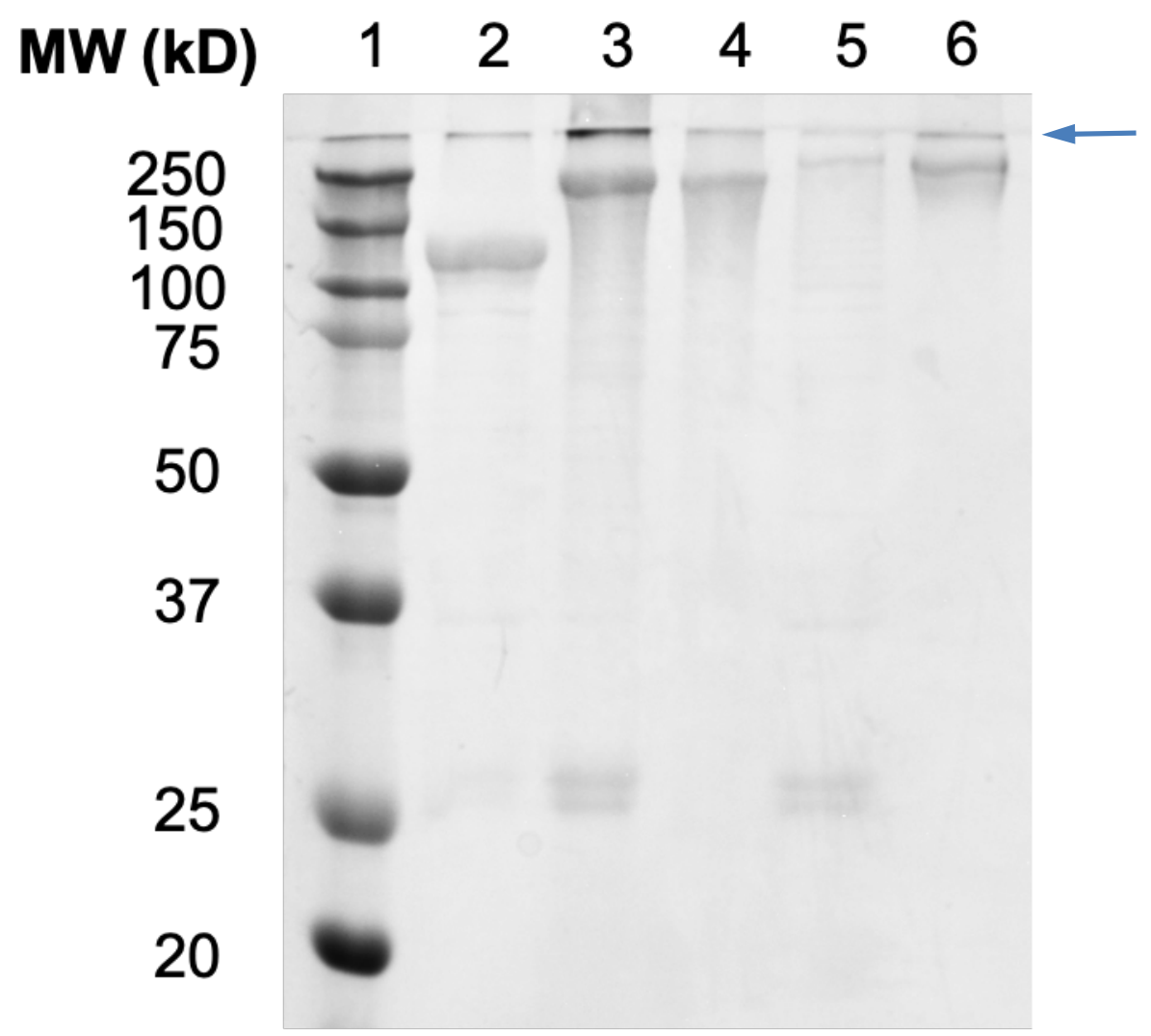

Fig S6. Coomassie blue-stained 10\% SDS-PAGE gel of purified high MW FGAILSS polymeric proteins. Lane 1, molecular weight marker with their sized labeled on the left. Lane 2, 48xFGAILSS protein purified using Ni-NTA affinity chromatography. Lane 3, 96xFGAILSS protein purified using Ni-NTA affinity chromatography. Lane 4, 96xFGAILSS protein from Lane 3 was further purified using size-exclusion chromatography. Lane 5, 128xFGAILSS protein purified using Ni-NTA affinity chromatography. Lane 6, 128xFGAILSS protein from Lane 5 was further purified using size-exclusion chromatography. The blue arrow indicates the border between stacking gel and resolving gel. 
(a)

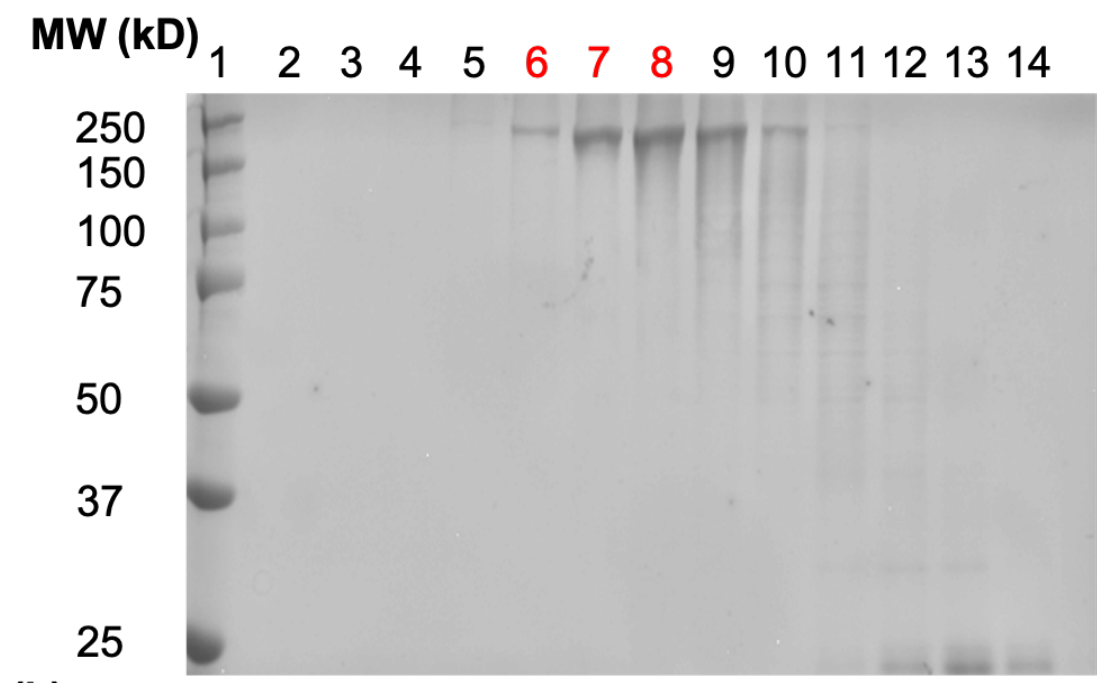

(b)

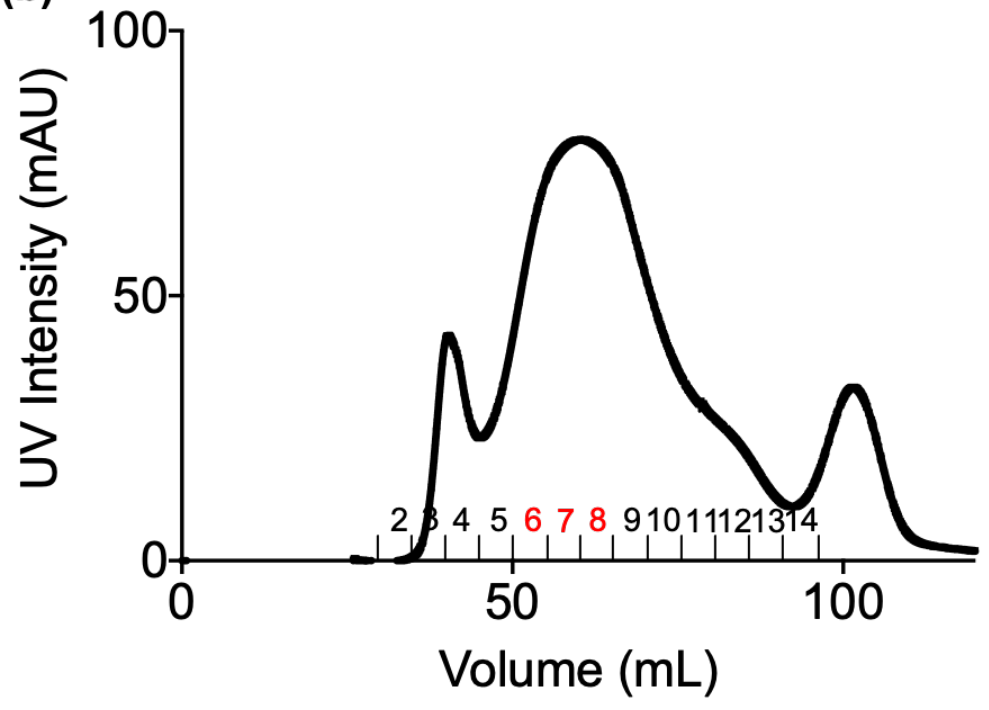

Fig S7. SEC purification of 96xFGAILSS protein. (a) 10\% SDS-PAGE gel of the elute protein fractions collected from SEC purification. (b) SEC protein elution spectrum. Proteins were detected by an UV detector at $280 \mathrm{~nm}$. Lane 1, molecular weight marker with their sized labeled on the left. Each of the rest lane corresponded to the part of spectrum labeled with the same number. The portions labeled in red were used for the preparation of protein dopes. 
(a)

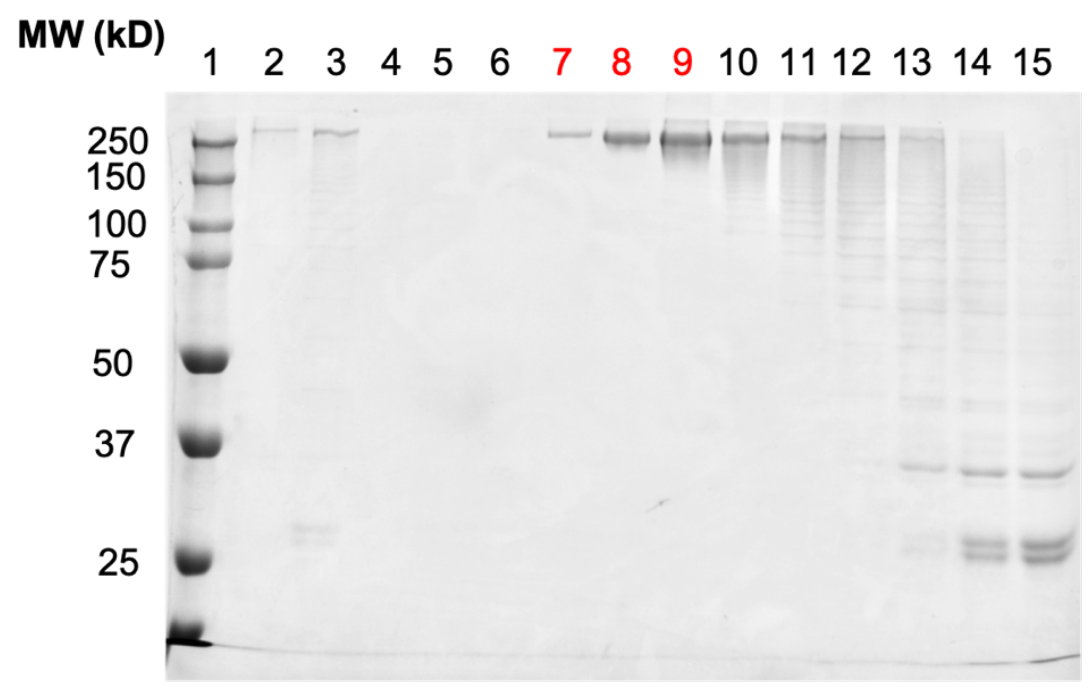

(b)

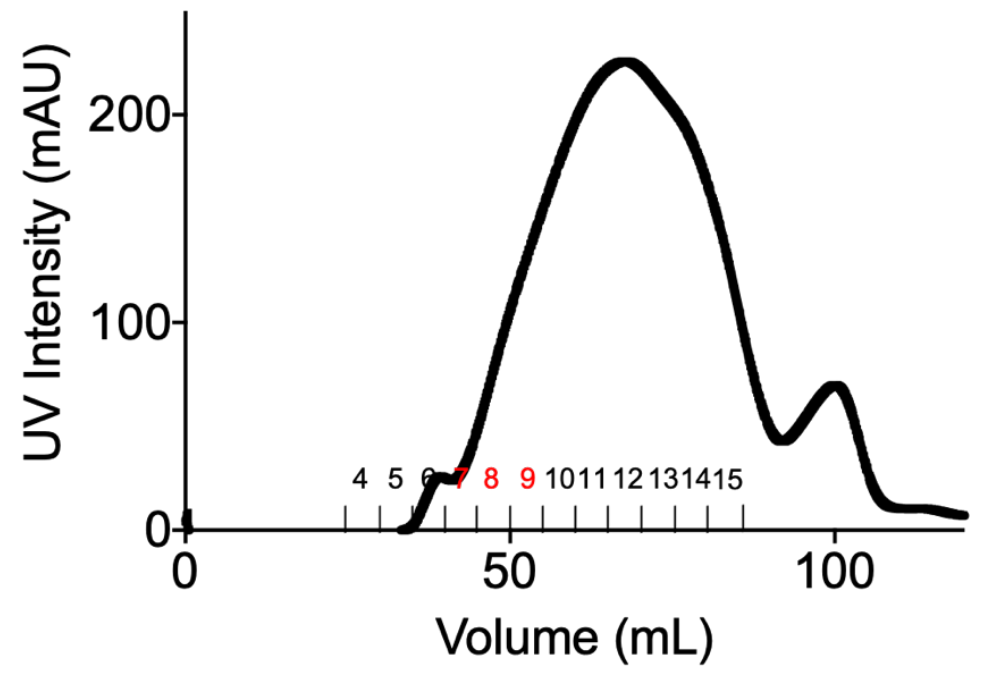

Fig S8. SEC purification of 128x FGAILSS hybrid protein. (a) 10\% SDS-PAGE gel of the elute protein fractions collected from SEC purification. (b) SEC protein elution spectrum. Proteins were detected by an UV detector at $280 \mathrm{~nm}$. Lane 1, molecular weight marker with their sized labeled on the left. Lane 2 and 3, elutions from Ni-NTA columns with 50mM and 300mM imidazole. Each of the rest lane corresponded to the part of spectrum within the same column. The portions labeled in red were used for the preparation of protein dopes. 


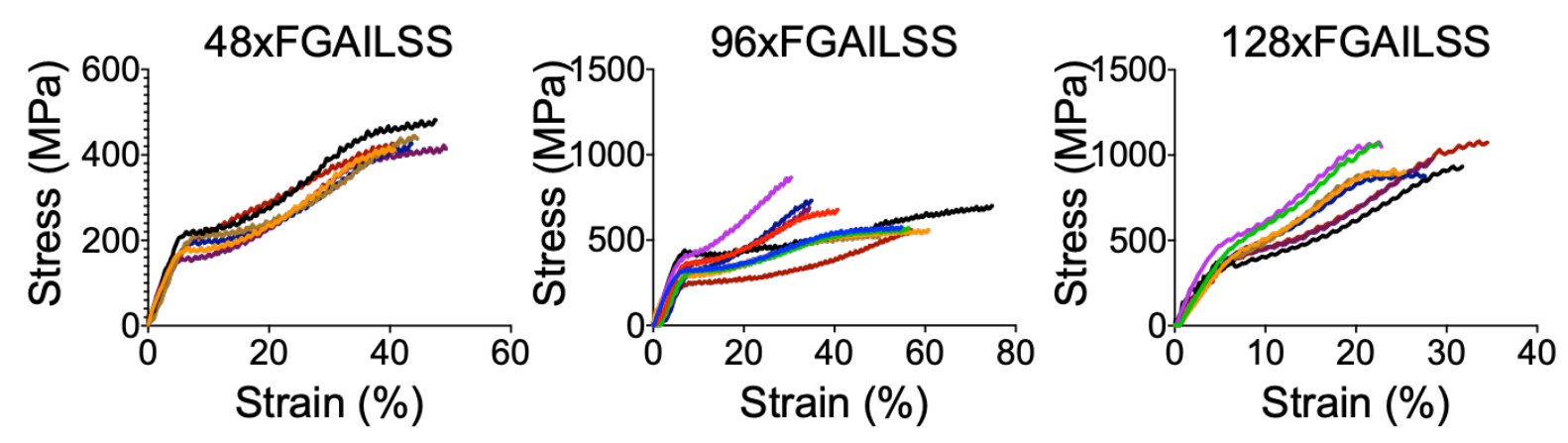

Fig S9. Stress-strain curves of high MW FGAILSS polymeric amyloid fibers. Each color represents an individual fiber. 
Table S1. Amyloid peptide sequences used in this study.

\begin{tabular}{|c|c|c|c|c|}
\hline & $\begin{array}{l}\text { Symmetry } \\
\text { Category }\end{array}$ & $\begin{array}{l}\text { Structural } \\
\text { Class }\end{array}$ & PDB ID & Origin \\
\hline GDVIEV & $\begin{array}{l}\text { Parallel- } \\
\text { Antifacial }\end{array}$ & Class 4 & 3SGS & $\alpha \mathrm{B}$-crystallin ${ }_{95-100}$ \\
\hline KLVFFAE & $\begin{array}{l}\text { Antiparallel- } \\
\text { Antifacial }\end{array}$ & Class 7 & 3OW9 & $\beta$-amyloid ${ }_{16-22}$ \\
\hline FGAILSS & $\begin{array}{l}\text { Antiparallel- } \\
\text { Equifacial }\end{array}$ & Class 6 & 5E61 & islet amyloid polypeptide $23-29$ \\
\hline
\end{tabular}


Table S2. Amino acid sequences used in polymeric amyloid proteins and the poly-alanine protein. Amyloid sequences in tandem repeats are in bold.

\begin{tabular}{|l|l|}
\hline & Amino acid sequence \\
\hline 1x KLVFFAE tandem repeat & GRGGLGGQGAGKLVFFAEGGAGQGGYGGLGSQG \\
\hline 1x FGAILSS tandem repeat & GRGGLGGQGAGFGAILSSGGAGQGGYGGLGSQG \\
\hline 1x GDVIEV tandem repeat & GRGGLGGQGAGGDVIEVGGAGQGGYGGLGSQG \\
\hline 1x poly-alanine tandem repeat & GRGGLGGQGAGAAAAAGGAGQGGYGGLGSQG \\
\hline
\end{tabular}


Table S3. Summary of amino acid sequences of all proteins used in this study.

\begin{tabular}{|c|c|}
\hline & 1x Sequence \\
\hline 16xGDVIEV & $\begin{array}{l}\text { MAKTKGTASGRGGLGGQGAGGDVIEVGGAGQGGYGGLGSQG } \\
\text { TSGRGGLGGQGAGGDVIEVGGAGQGGYGGLGSQGTSGRGGLG } \\
\text { GQGAGGDVIEVGGAGQGGYGGLGSQGTSGRGGLGGQGAGGD } \\
\text { VIEVGGAGQGGYGGLGSQGTSGRGGLGGQGAGGDVIEVGGAG } \\
\text { QGGYGGLGSQGTSGRGGLGGQGAGGDVIEVGGAGQGGYGGL } \\
\text { GSQGTSGRGGLGGQGAGGDVIEVGGAGQGGYGGLGSQGTSGR } \\
\text { GGLGGQGAGGDVIEVGGAGQGGYGGLGSQGTSGRGGLGGQG } \\
\text { AGGDVIEVGGAGQGGYGGLGSQGTSGRGGLGGQGAGGDVIEV } \\
\text { GGAGQGGYGGLGSQGTSGRGGLGGQGAGGDVIEVGGAGQGG } \\
\text { YGGLGSQGTSGRGGLGGQGAGGDVIEVGGAGQGGYGGLGSQ } \\
\text { GTSGRGGLGGQGAGGDVIEVGGAGQGGYGGLGSQGTSGRGGL } \\
\text { GGQGAGGDVIEVGGAGQGGYGGLGSQGTSGRGGLGGQGAGG } \\
\text { DVIEVGGAGQGGYGGLGSQGTSGRGGLGGQGAGGDVIEVGGA } \\
\text { GQGGYGGLGSQGTSSGHHHHHHHHHH }\end{array}$ \\
\hline 16xKLVFFAE & $\begin{array}{l}\text { MAKTKGTASGRGGLGGQGAGKLVFFAEGGAGQGGYGGLGSQ } \\
\text { GTSGRGGLGGQGAGKLVFFAEGGAGQGGYGGLGSQGTSGRG } \\
\text { GLGGQGAGKLVFFAEGGAGQGGYGGLGSQGTSGRGGLGGQG } \\
\text { AGKLVFFAEGGAGQGGYGGLGSQGTSGRGGLGGQGAGKLVFF } \\
\text { AEGGAGQGGYGGLGSQGTSGRGGLGGQGAGKLVFFAEGGAG } \\
\text { QGGYGGLGSQGTSGRGGLGGQGAGKLVFFAEGGAGQGGYGG } \\
\text { LGSQGTSGRGGLGGQGAGKLVFFAEGGAGQGGYGGLGSQGTS } \\
\text { GRGGLGGQGAGKLVFFAEGGAGQGGYGGLGSQGTSGRGGLG } \\
\text { GQGAGKLVFFAEGGAGQGGYGGLGSQGTSGRGGLGGQGAGK } \\
\text { LVFFAEGGAGQGGYGGLGSQGTSGRGGLGGQGAGKLVFFAEG } \\
\text { GAGQGGYGGLGSQGTSGRGGLGGQGAGKLVFFAEGGAGQGG } \\
\text { YGGLGSQGTSGRGGLGGQGAGKLVFFAEGGAGQGGYGGLGS } \\
\text { QGTSGRGGLGGQGAGKLVFFAEGGAGQGGYGGLGSQGTSGR } \\
\text { GGLGGQGAGKLVFFAEGGAGQGGYGGLGSQGTSSGHHHHHH } \\
\text { HHHH }\end{array}$ \\
\hline 16xFGAILSS & $\begin{array}{l}\text { MAKTKGTASGRGGLGGQGAGFGAILSSGGAGQGGYGGLGSQG } \\
\text { TSGRGGLGGQGAGFGAILSSGGAGQGGYGGLGSQGTSGRGGL } \\
\text { GGQGAGFGAILSSGGAGQGGYGGLGSQGTSGRGGLGGQGAGF } \\
\text { GAILSSGGAGQGGYGGLGSQGTSGRGGLGGQGAGFGAILSSGG } \\
\text { AGQGGYGGLGSQGTSGRGGLGGQGAGFGAILSSGGAGQGGYG } \\
\text { GLGSQGTSGRGGLGGQGAGFGAILSSGGAGQGGYGGLGSQGT } \\
\text { SGRGGLGGQGAGFGAILSSGGAGQGGYGGLGSQGTSGRGGLG } \\
\text { GQGAGFGAILSSGGAGQGGYGGLGSQGTSGRGGLGGQGAGFG }\end{array}$ \\
\hline
\end{tabular}




\begin{tabular}{|c|c|}
\hline & $\begin{array}{l}\text { AILSSGGAGQGGYGGLGSQGTSGRGGLGGQGAGFGAILSSGGA } \\
\text { GQGGYGGLGSQGTSGRGGLGGQGAGFGAILSSGGAGQGGYGG } \\
\text { LGSQGTSGRGGLGGQGAGFGAILSSGGAGQGGYGGLGSQGTS } \\
\text { GRGGLGGQGAGFGAILSSGGAGQGGYGGLGSQGTSGRGGLGG } \\
\text { QGAGFGAILSSGGAGQGGYGGLGSQGTSGRGGLGGQGAGFGA } \\
\text { ILSSGGAGQGGYGGLGSQGTSSGHHHHHHHHHH }\end{array}$ \\
\hline $\begin{array}{l}\text { 16xRecombinant } \\
\text { silk }\end{array}$ & $\begin{array}{l}\text { MAKTKGTASGRGGLGGQGAGAAAAAGGAGQGGYGGLGSQG } \\
\text { TSGRGGLGGQGAGAAAAAGGAGQGGYGGLGSQGTSGRGGLG } \\
\text { GQGAGAAAAAGGAGQGGYGGLGSQGTSGRGGLGGQGAGAA } \\
\text { AAAGGAGQGGYGGLGSQGTSGRGGLGGQGAGAAAAAGGAG } \\
\text { QGGYGGLGSQGTSGRGGLGGQGAGAAAAAGGAGQGGYGGL } \\
\text { GSQGTSGRGGLGGQGAGAAAAAGGAGQGGYGGLGSQGTSGR } \\
\text { GGLGGQGAGAAAAAGGAGQGGYGGLGSQGTSGRGGLGGQG } \\
\text { AGAAAAAGGAGQGGYGGLGSQGTSGRGGLGGQGAGAAAAA } \\
\text { GGAGQGGYGGLGSQGTSGRGGLGGQGAGAAAAAGGAGQGG } \\
\text { YGGLGSQGTSGRGGLGGQGAGAAAAAGGAGQGGYGGLGSQG } \\
\text { TSGRGGLGGQGAGAAAAAGGAGQGGYGGLGSQGTSGRGGLG } \\
\text { GQGAGAAAAAGGAGQGGYGGLGSQGTSGRGGLGGQGAGAA } \\
\text { AAAGGAGQGGYGGLGSQGTSGRGGLGGQGAGAAAAAGGAG } \\
\text { QGGYGGLGSQGTSSGHHHHHHHHHH }\end{array}$ \\
\hline 48xFGAILSS & $\begin{array}{l}\text { MAKTKGTASGRGGLGGQGAGFGAILSSGGAGQGGYGGLGSQG } \\
\text { TSGRGGLGGQGAGFGAILSSGGAGQGGYGGLGSQGTSGRGGL } \\
\text { GGQGAGFGAILSSGGAGQGGYGGLGSQGTSGRGGLGGQGAGF } \\
\text { GAILSSGGAGQGGYGGLGSQGTSGRGGLGGQGAGFGAILSSGG } \\
\text { AGQGGYGGLGSQGTSGRGGLGGQGAGFGAILSSGGAGQGGYG } \\
\text { GLGSQGTSGRGGLGGQGAGFGAILSSGGAGQGGYGGLGSQGT } \\
\text { SGRGGLGGQGAGFGAILSSGGAGQGGYGGLGSQGTSGRGGLG } \\
\text { GQGAGFGAILSSGGAGQGGYGGLGSQGTSGRGGLGGQGAGFG } \\
\text { AILSSGGAGQGGYGGLGSQGTSGRGGLGGQGAGFGAILSSGGA } \\
\text { GQGGYGGLGSQGTSGRGGLGGQGAGFGAILSSGGAGQGGYGG } \\
\text { LGSQGTSGRGGLGGQGAGFGAILSSGGAGQGGYGGLGSQGTS } \\
\text { GRGGLGGQGAGFGAILSSGGAGQGGYGGLGSQGTSGRGGLGG } \\
\text { QGAGFGAILSSGGAGQGGYGGLGSQGTSGRGGLGGQGAGFGA } \\
\text { ILSSGGAGQGGYGGLGSQGTSGRGGLGGQGAGFGAILSSGGAG } \\
\text { QGGYGGLGSQGTSGRGGLGGQGAGFGAILSSGGAGQGGYGGL } \\
\text { GSQGTSGRGGLGGQGAGFGAILSSGGAGQGGYGGLGSQGTSG } \\
\text { RGGLGGQGAGFGAILSSGGAGQGGYGGLGSQGTSGRGGLGGQ } \\
\text { GAGFGAILSSGGAGQGGYGGLGSQGTSGRGGLGGQGAGFGAIL } \\
\text { SSGGAGQGGYGGLGSQGTSGRGGLGGQGAGFGAILSSGGAGQ } \\
\text { GGYGGLGSQGTSGRGGLGGQGAGFGAILSSGGAGQGGYGGLG } \\
\text { SQGTSGRGGLGGQGAGFGAILSSGGAGQGGYGGLGSQGTSGR }\end{array}$ \\
\hline
\end{tabular}




\begin{tabular}{|c|c|}
\hline & $\begin{array}{l}\text { GGLGGQGAGFGAILSSGGAGQGGYGGLGSQGTSGRGGLGGQG } \\
\text { AGFGAILSSGGAGQGGYGGLGSQGTSGRGGLGGQGAGFGAILS } \\
\text { SGGAGQGGYGGLGSQGTSGRGGLGGQGAGFGAILSSGGAGQG } \\
\text { GYGGLGSQGTSGRGGLGGQGAGFGAILSSGGAGQGGYGGLGS } \\
\text { QGTSGRGGLGGQGAGFGAILSSGGAGQGGYGGLGSQGTSGRG } \\
\text { GLGGQGAGFGAILSSGGAGQGGYGGLGSQGTSGRGGLGGQGA } \\
\text { GFGAILSSGGAGQGGYGGLGSQGTSGRGGLGGQGAGFGAILSS } \\
\text { GGAGQGGYGGLGSQGTSGRGGLGGQGAGFGAILSSGGAGQGG } \\
\text { YGGLGSQGTSGRGGLGGQGAGFGAILSSGGAGQGGYGGLGSQ } \\
\text { GTSGRGGLGGQGAGFGAILSSGGAGQGGYGGLGSQGTSGRGG } \\
\text { LGGQGAGFGAILSSGGAGQGGYGGLGSQGTSGRGGLGGQGAG } \\
\text { FGAILSSGGAGQGGYGGLGSQGTSGRGGLGGQGAGFGAILSSG } \\
\text { GAGQGGYGGLGSQGTSGRGGLGGQGAGFGAILSSGGAGQGGY } \\
\text { GGLGSQGTSGRGGLGGQGAGFGAILSSGGAGQGGYGGLGSQG } \\
\text { TSGRGGLGGQGAGFGAILSSGGAGQGGYGGLGSQGTSGRGGL } \\
\text { GGQGAGFGAILSSGGAGQGGYGGLGSQGTSGRGGLGGQGAGF } \\
\text { GAILSSGGAGQGGYGGLGSQGTSGRGGLGGQGAGFGAILSSGG } \\
\text { AGQGGYGGLGSQGTSGRGGLGGQGAGFGAILSSGGAGQGGYG } \\
\text { GLGSQGTSGRGGLGGQGAGFGAILSSGGAGQGGYGGLGSQGT } \\
\text { SSGHHHHHHHHHH }\end{array}$ \\
\hline 96xFGAILSS & $\begin{array}{l}\text { MAKTKGTASGRGGLGGQGAGFGAILSSGGAGQGGYGGLGSQG } \\
\text { TSGRGGLGGQGAGFGAILSSGGAGQGGYGGLGSQGTSGRGGL } \\
\text { GGQGAGFGAILSSGGAGQGGYGGLGSQGTSGRGGLGGQGAGF } \\
\text { GAILSSGGAGQGGYGGLGSQGTSGRGGLGGQGAGFGAILSSGG } \\
\text { AGQGGYGGLGSQGTSGRGGLGGQGAGFGAILSSGGAGQGGYG } \\
\text { GLGSQGTSGRGGLGGQGAGFGAILSSGGAGQGGYGGLGSQGT } \\
\text { SGRGGLGGQGAGFGAILSSGGAGQGGYGGLGSQGTSGRGGLG } \\
\text { GQGAGFGAILSSGGAGQGGYGGLGSQGTSGRGGLGGQGAGFG } \\
\text { AILSSGGAGQGGYGGLGSQGTSGRGGLGGQGAGFGAILSSGGA } \\
\text { GQGGYGGLGSQGTSGRGGLGGQGAGFGAILSSGGAGQGGYGG } \\
\text { LGSQGTSGRGGLGGQGAGFGAILSSGGAGQGGYGGLGSQGTS } \\
\text { GRGGLGGQGAGFGAILSSGGAGQGGYGGLGSQGTSGRGGLGG } \\
\text { QGAGFGAILSSGGAGQGGYGGLGSQGTSGRGGLGGQGAGFGA } \\
\text { ILSSGGAGQGGYGGLGSQGTSGRGGLGGQGAGFGAILSSGGAG } \\
\text { QGGYGGLGSQGTSGRGGLGGQGAGFGAILSSGGAGQGGYGGL } \\
\text { GSQGTSGRGGLGGQGAGFGAILSSGGAGQGGYGGLGSQGTSG } \\
\text { RGGLGGQGAGFGAILSSGGAGQGGYGGLGSQGTSGRGGLGGQ } \\
\text { GAGFGAILSSGGAGQGGYGGLGSQGTSGRGGLGGQGAGFGAIL } \\
\text { SSGGAGQGGYGGLGSQGTSGRGGLGGQGAGFGAILSSGGAGQ } \\
\text { GGYGGLGSQGTSGRGGLGGQGAGFGAILSSGGAGQGGYGGLG } \\
\text { SQGTSGRGGLGGQGAGFGAILSSGGAGQGGYGGLGSQGTSGR } \\
\text { GGLGGQGAGFGAILSSGGAGQGGYGGLGSQGTSGRGGLGGQG } \\
\text { AGFGAILSSGGAGQGGYGGLGSQGTSGRGGLGGQGAGFGAILS } \\
\text { SGGAGQGGYGGLGSQGTSGRGGLGGQGAGFGAILSSGGAGQG }\end{array}$ \\
\hline
\end{tabular}


GYGGLGSQGTSGRGGLGGQGAGFGAILSSGGAGQGGYGGLGS QGTSGRGGLGGQGAGFGAILSSGGAGQGGYGGLGSQGTSGRG GLGGQGAGFGAILSSGGAGQGGYGGLGSQGTSGRGGLGGQGA GFGAILSSGGAGQGGYGGLGSQGTSGRGGLGGQGAGFGAILSS GGAGQGGYGGLGSQGTSGRGGLGGQGAGFGAILSSGGAGQGG YGGLGSQGTSGRGGLGGQGAGFGAILSSGGAGQGGYGGLGSQ GTSGRGGLGGQGAGFGAILSSGGAGQGGYGGLGSQGTSGRGG LGGQGAGFGAILSSGGAGQGGYGGLGSQGTSGRGGLGGQGAG FGAILSSGGAGQGGYGGLGSQGTSGRGGLGGQGAGFGAILSSG GAGQGGYGGLGSQGTSGRGGLGGQGAGFGAILSSGGAGQGGY GGLGSQGTSGRGGLGGQGAGFGAILSSGGAGQGGYGGLGSQG TSGRGGLGGQGAGFGAILSSGGAGQGGYGGLGSQGTSGRGGL GGQGAGFGAILSSGGAGQGGYGGLGSQGTSGRGGLGGQGAGF GAILSSGGAGQGGYGGLGSQGTSGRGGLGGQGAGFGAILSSGG AGQGGYGGLGSQGTSGRGGLGGQGAGFGAILSSGGAGQGGYG GLGSQGTSGRGGLGGQGAGFGAILSSGGAGQGGYGGLGSQGT SGRGGLGGQGAGFGAILSSGGAGQGGYGGLGSQGTSGRGGLG GQGAGFGAILSSGGAGQGGYGGLGSQGTSGRGGLGGQGAGFG AILSSGGAGQGGYGGLGSQGTSGRGGLGGQGAGFGAILSSGGA GQGGYGGLGSQGTSGRGGLGGQGAGFGAILSSGGAGQGGYGG LGSQGTSGRGGLGGQGAGFGAILSSGGAGQGGYGGLGSQGTS GRGGLGGQGAGFGAILSSGGAGQGGYGGLGSQGTSGRGGLGG QGAGFGAILSSGGAGQGGYGGLGSQGTSGRGGLGGQGAGFGA ILSSGGAGQGGYGGLGSQGTSGRGGLGGQGAGFGAILSSGGAG QGGYGGLGSQGTSGRGGLGGQGAGFGAILSSGGAGQGGYGGL GSQGTSGRGGLGGQGAGFGAILSSGGAGQGGYGGLGSQGTSG RGGLGGQGAGFGAILSSGGAGQGGYGGLGSQGTSGRGGLGGQ GAGFGAILSSGGAGQGGYGGLGSQGTSGRGGLGGQGAGFGAIL SSGGAGQGGYGGLGSQGTSGRGGLGGQGAGFGAILSSGGAGQ GGYGGLGSQGTSGRGGLGGQGAGFGAILSSGGAGQGGYGGLG SQGTSGRGGLGGQGAGFGAILSSGGAGQGGYGGLGSQGTSGR GGLGGQGAGFGAILSSGGAGQGGYGGLGSQGTSGRGGLGGQG AGFGAILSSGGAGQGGYGGLGSQGTSGRGGLGGQGAGFGAILS SGGAGQGGYGGLGSQGTSGRGGLGGQGAGFGAILSSGGAGQG GYGGLGSQGTSGRGGLGGQGAGFGAILSSGGAGQGGYGGLGS QGTSGRGGLGGQGAGFGAILSSGGAGQGGYGGLGSQGTSGRG GLGGQGAGFGAILSSGGAGQGGYGGLGSQGTSGRGGLGGQGA GFGAILSSGGAGQGGYGGLGSQGTSGRGGLGGQGAGFGAILSS GGAGQGGYGGLGSQGTSGRGGLGGQGAGFGAILSSGGAGQGG YGGLGSQGTSGRGGLGGQGAGFGAILSSGGAGQGGYGGLGSQ GTSGRGGLGGQGAGFGAILSSGGAGQGGYGGLGSQGTSGRGG LGGQGAGFGAILSSGGAGQGGYGGLGSQGTSGRGGLGGQGAG FGAILSSGGAGQGGYGGLGSQGTSGRGGLGGQGAGFGAILSSG GAGQGGYGGLGSQGTSGRGGLGGQGAGFGAILSSGGAGQGGY GGLGSQGTSGRGGLGGQGAGFGAILSSGGAGQGGYGGLGSQG TSGRGGLGGQGAGFGAILSSGGAGQGGYGGLGSQGTSGRGGL 


\begin{tabular}{|c|c|}
\hline & $\begin{array}{l}\text { GGQGAGFGAILSSGGAGQGGYGGLGSQGTSGRGGLGGQGAGF } \\
\text { GAILSSGGAGQGGYGGLGSQGTSGRGGLGGQGAGFGAILSSGG } \\
\text { AGQGGYGGLGSQGTSGRGGLGGQGAGFGAILSSGGAGQGGYG } \\
\text { GLGSQGTSGRGGLGGQGAGFGAILSSGGAGQGGYGGLGSQGT } \\
\text { SGRGGLGGQGAGFGAILSSGGAGQGGYGGLGSQGTSGRGGLG } \\
\text { GQGAGFGAILSSGGAGQGGYGGLGSQGTSGRGGLGGQGAGFG } \\
\text { AILSSGGAGQGGYGGLGSQGTSGRGGLGGQGAGFGAILSSGGA } \\
\text { GQGGYGGLGSQGTSGRGGLGGQGAGFGAILSSGGAGQGGYGG } \\
\text { LGSQGTSGRGGLGGQGAGFGAILSSGGAGQGGYGGLGSQGTS } \\
\text { GRGGLGGQGAGFGAILSSGGAGQGGYGGLGSQGTSSGHHHHH } \\
\text { HHHHH }\end{array}$ \\
\hline 128xFGAILSS & $\begin{array}{l}\text { MAKTKGTASGRGGLGGQGAGFGAILSSGGAGQGGYGGLGSQG } \\
\text { TSGRGGLGGQGAGFGAILSSGGAGQGGYGGLGSQGTSGRGGL } \\
\text { GGQGAGFGAILSSGGAGQGGYGGLGSQGTSGRGGLGGQGAGF } \\
\text { GAILSSGGAGQGGYGGLGSQGTSGRGGLGGQGAGFGAILSSGG } \\
\text { AGQGGYGGLGSQGTSGRGGLGGQGAGFGAILSSGGAGQGGYG } \\
\text { GLGSQGTSGRGGLGGQGAGFGAILSSGGAGQGGYGGLGSQGT } \\
\text { SGRGGLGGQGAGFGAILSSGGAGQGGYGGLGSQGTSGRGGLG } \\
\text { GQGAGFGAILSSGGAGQGGYGGLGSQGTSGRGGLGGQGAGFG } \\
\text { AILSSGGAGQGGYGGLGSQGTSGRGGLGGQGAGFGAILSSGGA } \\
\text { GQGGYGGLGSQGTSGRGGLGGQGAGFGAILSSGGAGQGGYGG } \\
\text { LGSQGTSGRGGLGGQGAGFGAILSSGGAGQGGYGGLGSQGTS } \\
\text { GRGGLGGQGAGFGAILSSGGAGQGGYGGLGSQGTSGRGGLGG } \\
\text { QGAGFGAILSSGGAGQGGYGGLGSQGTSGRGGLGGQGAGFGA } \\
\text { ILSSGGAGQGGYGGLGSQGTSGRGGLGGQGAGFGAILSSGGAG } \\
\text { QGGYGGLGSQGTSGRGGLGGQGAGFGAILSSGGAGQGGYGGL } \\
\text { GSQGTSGRGGLGGQGAGFGAILSSGGAGQGGYGGLGSQGTSG } \\
\text { RGGLGGQGAGFGAILSSGGAGQGGYGGLGSQGTSGRGGLGGQ } \\
\text { GAGFGAILSSGGAGQGGYGGLGSQGTSGRGGLGGQGAGFGAIL } \\
\text { SSGGAGQGGYGGLGSQGTSGRGGLGGQGAGFGAILSSGGAGQ } \\
\text { GGYGGLGSQGTSGRGGLGGQGAGFGAILSSGGAGQGGYGGLG } \\
\text { SQGTSGRGGLGGQGAGFGAILSSGGAGQGGYGGLGSQGTSGR } \\
\text { GGLGGQGAGFGAILSSGGAGQGGYGGLGSQGTSGRGGLGGQG } \\
\text { AGFGAILSSGGAGQGGYGGLGSQGTSGRGGLGGQGAGFGAILS } \\
\text { SGGAGQGGYGGLGSQGTSGRGGLGGQGAGFGAILSSGGAGQG } \\
\text { GYGGLGSQGTSGRGGLGGQGAGFGAILSSGGAGQGGYGGLGS } \\
\text { QGTSGRGGLGGQGAGFGAILSSGGAGQGGYGGLGSQGTSGRG } \\
\text { GLGGQGAGFGAILSSGGAGQGGYGGLGSQGTSGRGGLGGQGA } \\
\text { GFGAILSSGGAGQGGYGGLGSQGTSGRGGLGGQGAGFGAILSS } \\
\text { GGAGQGGYGGLGSQGTSGRGGLGGQGAGFGAILSSGGAGQGG } \\
\text { YGGLGSQGTSGRGGLGGQGAGFGAILSSGGAGQGGYGGLGSQ } \\
\text { GTSGRGGLGGQGAGFGAILSSGGAGQGGYGGLGSQGTSGRGG } \\
\text { LGGQGAGFGAILSSGGAGQGGYGGLGSQGTSGRGGLGGQGAG } \\
\text { FGAILSSGGAGQGGYGGLGSQGTSGRGGLGGQGAGFGAILSSG }\end{array}$ \\
\hline
\end{tabular}




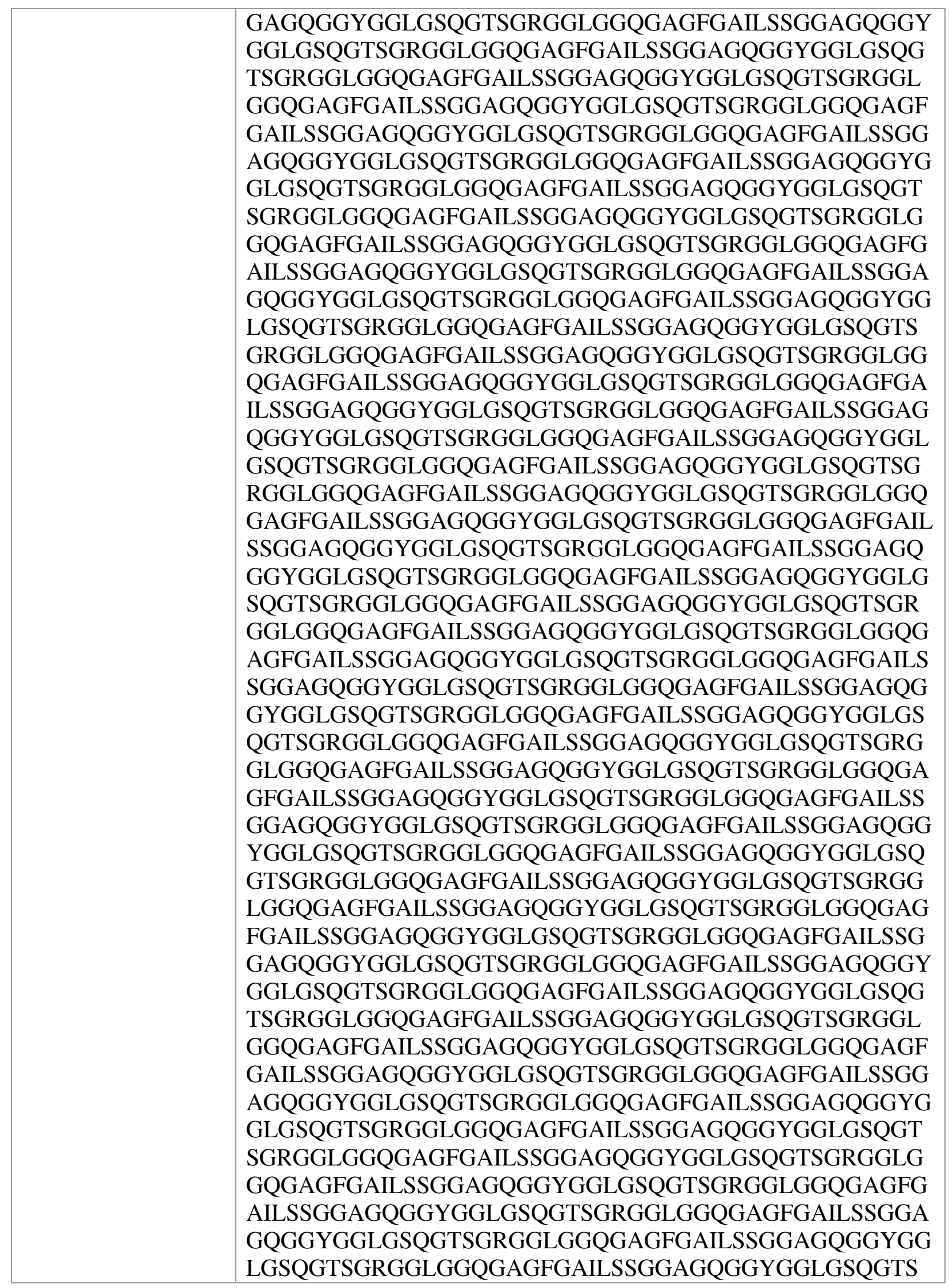




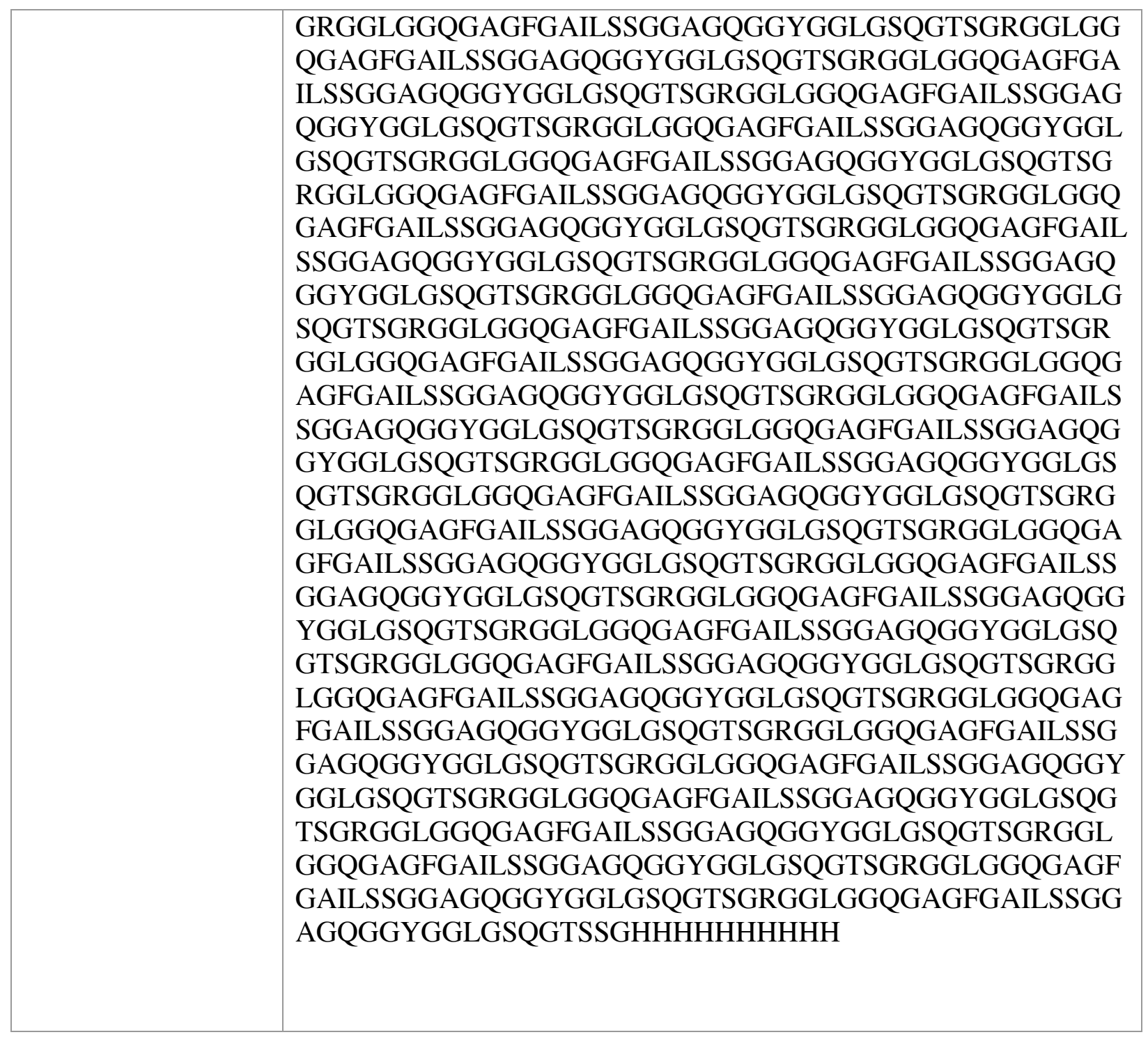


Table S4. Summary of protein yield and productivity for all polymeric amyloid proteins used in the study. Expression level was calculated as the percent of overexpressed amyloid protein out of the total proteome of the corresponding E. coli strain as measured from densitometric analysis of Coomassie Blue-stained SDS-PAGE gels.

\begin{tabular}{|l|l|l|}
\hline & Yield (\%) & Productivity (mg purified/g dry cell) \\
\hline 16xKLVFFAE & 16 & 3.3 \\
\hline 16xFGAILSS & 27 & 5.2 \\
\hline 16xGDVIEV & 20 & 4.9 \\
\hline 48xFGAILSS & 6.7 & 3.0 \\
\hline 96xFGAILSS & 6.3 & 1.4 \\
\hline 128xFGAILSS & 4.5 & 1.5 \\
\hline
\end{tabular}


Table S5. Diameters of 16xKLVFFAE fibers.

\begin{tabular}{|l|l|l|l|l|}
\hline & D1 $(\boldsymbol{\mu m}) *$ & D2 $(\boldsymbol{\mu m})^{*}$ & D3 $(\boldsymbol{\mu m})^{*}$ & $\begin{array}{l}\text { Average } \\
\text { diameter }(\boldsymbol{\mu m})\end{array}$ \\
\hline KLV-1 & 14 & 15 & 14 & 14 \\
\hline KLV-2 & 22 & 19 & 20 & 20 \\
\hline KLV-4 & 20 & 19 & 21 & 20 \\
\hline KLV-5 & 21 & 19 & 18 & 19 \\
\hline KLV-6 & 20 & 23 & 22 & 22 \\
\hline KLV-7 & 15 & 19 & 22 & 17 \\
\hline KLV-8 & 20 & 17 & 20 & 17 \\
\hline KLV-9 & 17 & 14 & 17 & 17 \\
\hline KLV-10 & 20 & 14 & 18 & 15 \\
\hline
\end{tabular}

*D1, D2, D3: Three independent diameter measurements of the same fiber at three different locations, see Methods for details. 
Table S6. Summary of mechanical properties of 16xKLVFFAE fibers.

\begin{tabular}{|c|c|c|c|c|c|}
\hline & $\begin{array}{l}\text { Ultimate } \\
\text { tensile stress } \\
\text { (MPa) }\end{array}$ & $\begin{array}{l}\text { Modulus } \\
\text { (GPa) }\end{array}$ & $\begin{array}{l}\text { Breaking } \\
\text { strain }(\%)\end{array}$ & $\begin{array}{l}\text { Toughness } \\
\left(\mathbf{M J}^{*} \mathbf{m}^{-3}\right)\end{array}$ & $\begin{array}{l}\text { Average } \\
\text { diameter } \\
(\mu \mathrm{m})\end{array}$ \\
\hline KLV-1 & 240 & 4.0 & 38 & 80 & 14 \\
\hline KLV-2 & 228 & 3.5 & 63 & 120 & 20 \\
\hline KLV-3 & 245 & 3.8 & 49 & 90 & 20 \\
\hline KLV-4 & 239 & 3.7 & 37 & 70 & 19 \\
\hline KLV-5 & 188 & 3.6 & 50 & 80 & 22 \\
\hline KLV-6 & 234 & 2.5 & 49 & 90 & 20 \\
\hline KLV-7 & 265 & 2.8 & 62 & 120 & 17 \\
\hline KLV-8 & 267 & 2.5 & 69 & 120 & 17 \\
\hline KLV-9 & 248 & 4.1 & 88 & 150 & 17 \\
\hline KLV-10 & 272 & 3.3 & 67 & 120 & 17 \\
\hline Average & 243 & 3.4 & 57 & 104 & 18 \\
\hline $\begin{array}{l}\text { Standard } \\
\text { Deviation }\end{array}$ & 24 & 0.6 & 16 & 26 & 2.3 \\
\hline
\end{tabular}


Table S7. Diameters of 16xFGAILSS fibers.

\begin{tabular}{|l|l|l|l|l|}
\hline & D1 $(\boldsymbol{\mu m})^{*}$ & D2 $(\boldsymbol{\mu m})^{*}$ & D3 $(\boldsymbol{\mu m})^{*}$ & Diameter $(\boldsymbol{\mu m})$ \\
\hline FGA-1 & 17 & 16 & 17 & 17 \\
\hline FGA-2 & 17 & 18 & 17 & 18 \\
\hline FGA-3 & 17 & 17 & 17 & 17 \\
\hline FGA-4 & 19 & 20 & 21 & 20 \\
\hline FGA-5 & 21 & 18 & 18 & 19 \\
\hline FGA-6 & 19 & 19 & 20 & 20 \\
\hline FGA-7 & 16 & 16 & 15 & 16 \\
\hline FGA-8 & 15 & 16 & 18 & 16 \\
\hline FGA-9 & 16 & 16 & 16 & 16 \\
\hline FGA-10 & 16 & 15 & 15 & 16 \\
\hline
\end{tabular}

*D1, D2, D3: Three independent diameter measurements of the same fiber at three different locations, see Methods for details. 
Table S8. Summary of mechanical properties of 16xFGAILSS silks in this study

\begin{tabular}{|c|c|c|c|c|c|}
\hline & $\begin{array}{l}\text { Ultimate } \\
\text { tensile stress } \\
\text { (MPa) }\end{array}$ & $\begin{array}{l}\text { Modulus } \\
\text { (GPa) }\end{array}$ & $\begin{array}{l}\text { Breaking } \\
\text { strain }(\%)\end{array}$ & $\begin{array}{l}\text { Toughness } \\
\left(\mathbf{M J} \mathbf{J}^{-3}\right)\end{array}$ & $\begin{array}{l}\text { Diameter } \\
(\mu \mathrm{m})\end{array}$ \\
\hline FGA-1 & 202 & 3.9 & 34 & 40 & 17 \\
\hline FGA-2 & 188 & 3.5 & 37 & 40 & 18 \\
\hline FGA-3 & 173 & 4.1 & 37 & 40 & 17 \\
\hline FGA-4 & 229 & 3.3 & 41 & 60 & 20 \\
\hline FGA-5 & 252 & 5.0 & 35 & 60 & 19 \\
\hline FGA-6 & 251 & 4.0 & 36 & 60 & 20 \\
\hline FGA-7 & 214 & 2.0 & 47 & 50 & 16 \\
\hline FGA-8 & 258 & 4.8 & 37 & 70 & 16 \\
\hline FGA-9 & 261 & 4.2 & 42 & 80 & 16 \\
\hline FGA-10 & 271 & 3.9 & 40 & 90 & 15 \\
\hline Average & 230 & 3.9 & 39 & 59 & 17 \\
\hline $\begin{array}{l}\text { Standard } \\
\text { Deviation }\end{array}$ & 34 & 0.8 & 4.0 & 17 & 2.0 \\
\hline
\end{tabular}


Table S9. Diameter Measurements of 16xGDVIEV silk.

\begin{tabular}{|l|l|l|l|l|}
\hline & D1 $(\boldsymbol{\mu m}) *$ & D2 $(\boldsymbol{\mu m})^{*}$ & D3 $(\boldsymbol{\mu m})^{*}$ & Diameter $(\boldsymbol{\mu m})$ \\
\hline GDV-1 & 11 & 12 & 12 & 12 \\
\hline GDV-2 & 11 & 12 & 12 & 12 \\
\hline GDV-3 & 11 & 11 & 11 & 11 \\
\hline GDV-5 & 11 & 11 & 12 & 11 \\
\hline GDV-6 & 11 & 11 & 12 & 12 \\
\hline GDV-7 & 9.9 & 11 & 10 & 10 \\
\hline GDV-8 & 10 & 9.9 & 10 & 10 \\
\hline GDV-9 & 10 & 10 & 10 & 10 \\
\hline GDV-10 & 10 & 9.6 & 10 & 10 \\
\hline
\end{tabular}

*D1, D2, D3: Three independent diameter measurements of the same fiber at three different locations, see Methods for details. 
Table S10. Summary of mechanical properties of 16xGDVIEV silks in this study

\begin{tabular}{|c|c|c|c|c|c|}
\hline & $\begin{array}{l}\text { Ultimate } \\
\text { tensile stress } \\
(\mathrm{MPa})\end{array}$ & $\begin{array}{l}\text { Modulus } \\
\text { (GPa) }\end{array}$ & $\begin{array}{l}\text { Breaking } \\
\text { strain (\%) }\end{array}$ & $\begin{array}{l}\text { Toughness } \\
\left(\mathbf{M J} \mathbf{J}^{*} \mathbf{m}^{-3}\right)\end{array}$ & $\begin{array}{l}\text { Diameter } \\
(\mu \mathrm{m})\end{array}$ \\
\hline GDV-1 & 296 & 6.0 & 24 & 40 & 12 \\
\hline GDV-2 & 258 & 3.2 & 35 & 60 & 12 \\
\hline GDV-3 & 322 & 6.7 & 22 & 50 & 11 \\
\hline GDV-4 & 311 & 7.3 & 23 & 50 & 11 \\
\hline GDV-5 & 279 & 4.7 & 28 & 50 & 12 \\
\hline GDV-6 & 228 & 5.3 & 45 & 70 & 10 \\
\hline GDV-7 & 208 & 5.8 & 31 & 70 & 10 \\
\hline GDV-8 & 198 & 4.9 & 30 & 70 & 10 \\
\hline GDV-9 & 394 & 4.4 & 33 & 60 & 10 \\
\hline GDV-10 & 309 & 5.1 & 38 & 70 & 10 \\
\hline Average & 280 & 5.3 & 31 & 59 & 11 \\
\hline $\begin{array}{l}\text { Standard } \\
\text { Deviation }\end{array}$ & 60 & 1.2 & 7.2 & 11 & 0.74 \\
\hline
\end{tabular}


Table S11. Diameter measurements of 16xpolyA silk.

\begin{tabular}{|l|l|l|l|l|}
\hline & D1 $(\boldsymbol{\mu m})^{*}$ & D2 $(\boldsymbol{\mu m})^{*}$ & D3 $(\boldsymbol{\mu m})^{*}$ & Diameter $(\boldsymbol{\mu m})$ \\
\hline polyA-1 & 20 & 19 & 18 & 19 \\
\hline polyA-2 & 15 & 16 & 18 & 17 \\
\hline polyA-3 & 18 & 18 & 17 & 17 \\
\hline polyA-4 & 16 & 17 & 18 & 17 \\
\hline polyA-5 & 17 & 16 & 16 & 16 \\
\hline polyA-6 & 19 & 18 & 18 & 18 \\
\hline
\end{tabular}

*D1, D2, D3: Three independent diameter measurements of the same fiber at three different locations, see Methods for details. 
Table S12. Summary of mechanical properties of 16xpolyA silks in this study

\begin{tabular}{|l|l|l|l|l|l|}
\hline & $\begin{array}{l}\text { Ultimate } \\
\text { tensile stress } \\
(\mathbf{M P a})\end{array}$ & $\begin{array}{l}\text { Modulus } \\
(\mathbf{G P a})\end{array}$ & $\begin{array}{l}\text { Breaking } \\
\text { strain }(\mathbf{\%})\end{array}$ & $\begin{array}{l}\text { Toughness } \\
\left(\mathbf{M J}^{*} \mathbf{m}^{-3}\right)\end{array}$ & $\begin{array}{l}\text { Diameter } \\
(\boldsymbol{\mu m})\end{array}$ \\
\hline polyA-1 & 74 & 1.9 & 40 & 25 & 19 \\
\hline polyA-2 & 98 & 3.4 & 54 & 40 & 17 \\
\hline polyA-3 & 77 & 2.4 & 43 & 26 & 17 \\
\hline polyA-4 & 104 & 3.1 & 59 & 50 & 17 \\
\hline polyA-5 & 73 & 2.6 & 84 & 50 & 18 \\
\hline polyA-6 & 78 & 2.1 & 87 & 50 & 17 \\
\hline Average & 84 & 2.6 & 61 & 40 & 1.1 \\
\hline $\begin{array}{l}\text { Standard } \\
\text { Deviation }\end{array}$ & 14 & 0.6 & 20 & 12 & \\
\hline
\end{tabular}


Table S13. Diameter measurements of 48xFGAILSS silk.

\begin{tabular}{|l|l|l|l|l|}
\hline & D1 $(\boldsymbol{\mu m})^{*}$ & D2 $(\boldsymbol{\mu m})^{*}$ & D3 $(\boldsymbol{\mu m})^{*}$ & Diameter $(\boldsymbol{\mu m})$ \\
\hline 48FGA-1 & 13 & 14 & 14 & 14 \\
\hline 48FGA-2 & 14 & 14 & 14 & 14 \\
\hline 48FGA-3 & 15 & 14 & 14 & 14 \\
\hline 48FGA-5 & 14 & 14 & 14 & 14 \\
\hline 48FGA-6 & 14 & 14 & 14 & 14 \\
\hline
\end{tabular}

*D1, D2, D3: Three independent diameter measurements of the same fiber at three different locations, see Methods for details. 
Table S14. Summary of mechanical properties of 48xFGAILSS hybrid proteins in this study

\begin{tabular}{|l|l|l|l|l|l|}
\hline & $\begin{array}{l}\text { Ultimate } \\
\text { tensile stress } \\
(\mathbf{M P a})\end{array}$ & $\begin{array}{l}\text { Modulus } \\
(\mathbf{G P a})\end{array}$ & $\begin{array}{l}\text { Breaking } \\
\text { strain }(\mathbf{\%})\end{array}$ & $\begin{array}{l}\text { Toughness } \\
\left(\mathbf{M J}^{*} \mathbf{m}^{-3}\right)\end{array}$ & $\begin{array}{l}\text { Diameter } \\
(\boldsymbol{\mu m})\end{array}$ \\
\hline 48FGA-1 & 432 & 3.7 & 41 & 120 & 14 \\
\hline 48FGA-2 & 422 & 4.7 & 49 & 130 & 14 \\
\hline 48FGA-3 & 428 & 3.7 & 43 & 110 & 14 \\
\hline 48FGA-4 & 445 & 3.8 & 43 & 120 & 14 \\
\hline 48FGA-5 & 482 & 4.9 & 47 & 160 & 14 \\
\hline 48FGA-6 & 417 & 4.1 & 40 & 100 & 14 \\
\hline Average & 438 & 4.2 & 44 & 123 & 0.27 \\
\hline $\begin{array}{l}\text { Standard } \\
\text { Deviation }\end{array}$ & 24 & 0.5 & 3.4 & 21 & \\
\hline
\end{tabular}


Table S15. Diameter measurements of 96xFGAILSS silk.

\begin{tabular}{|l|l|l|l|l|}
\hline & D1 $(\boldsymbol{\mu m}) *$ & D2 $(\boldsymbol{\mu m})^{*}$ & D3 $(\boldsymbol{\mu m})^{*}$ & Diameter $(\boldsymbol{\mu m})$ \\
\hline 96FGA-1 & 9.6 & 10 & 10 & 10 \\
\hline 96FGA-2 & 9.5 & 10 & 10 & 10 \\
\hline 96FGA-3 & 10 & 10 & 11 & 11 \\
\hline 96FGA-5 & 11 & 11 & 11 & 11 \\
\hline 96FGA-6 & 12 & 11 & 12 & 11 \\
\hline 96FGA-7 & 8.4 & 13 & 14 & 13 \\
\hline 96FGA-8 & 13 & 9.4 & 9.4 & 9.1 \\
\hline 96FGA-9 & 11 & 13 & 14 & 13 \\
\hline 96FGA-10 & 13 & 11 & 11 & 13 \\
\hline
\end{tabular}

*D1, D2, D3: Three independent diameter measurements of the same fiber at three different locations, see Methods for details. 
Table S16. Summary of mechanical properties of 96xFGAILSS proteins in this study.

\begin{tabular}{|c|c|c|c|c|c|}
\hline & $\begin{array}{l}\text { Ultimate } \\
\text { tensile stress } \\
\text { (MPa) }\end{array}$ & $\begin{array}{l}\text { Modulus } \\
\text { (GPa) }\end{array}$ & $\begin{array}{l}\text { Breaking } \\
\text { strain }(\%)\end{array}$ & $\begin{array}{l}\text { Toughness } \\
\left(\mathrm{MJ}^{*} \mathbf{m}^{-3}\right)\end{array}$ & $\begin{array}{l}\text { Diameter } \\
(\boldsymbol{\mu m})\end{array}$ \\
\hline 96FGA-1 & 560 & 4.7 & 56 & 190 & 10 \\
\hline 96FGA-2 & 689 & 7.0 & 33 & 140 & 10 \\
\hline 96FGA-3 & 731 & 6.8 & 32 & 140 & 11 \\
\hline 96FGA-4 & 533 & 6.2 & 48 & 200 & 11 \\
\hline 96FGA-5 & 700 & 10.0 & 74 & 400 & 11 \\
\hline 96FGA-6 & 558 & 7.2 & 61 & 260 & 13 \\
\hline 96FGA-7 & 866 & 9.5 & 30 & 160 & 9.1 \\
\hline 96FGA-8 & 571 & 7.1 & 55 & 230 & 13 \\
\hline 96FGA-9 & 678 & 7.6 & 39 & 190 & 11 \\
\hline 96FGA-10 & 577 & 7.1 & 53 & 230 & 13 \\
\hline Average & 646 & 7.3 & 48 & 214 & 11 \\
\hline $\begin{array}{l}\text { Standard } \\
\text { Deviation }\end{array}$ & 105 & 1.5 & 14 & 76 & 1.4 \\
\hline
\end{tabular}


Table S17. Diameter measurements of 128xFGAILSS silk.

\begin{tabular}{|l|l|l|l|l|}
\hline & D1 $(\boldsymbol{\mu m}) *$ & D2 $(\boldsymbol{\mu m})^{*}$ & D3 $(\boldsymbol{\mu m})^{*}$ & Diameter $(\boldsymbol{\mu m})$ \\
\hline 128FGA-1 & 8.8 & 9.1 & 9.4 & 9.1 \\
\hline 128FGA-2 & 9.9 & 9.4 & 9.5 & 9.6 \\
\hline 128FGAA-4 & 8.8 & 9.4 & 9.1 & 9.1 \\
\hline 128FGA-5 & 9.2 & 8.8 & 8.8 & 8.8 \\
\hline 128FGA-6 & 8.8 & 9.6 & 9.5 & 9.4 \\
\hline 128FGA-7 & 8.5 & 8.4 & 9.1 & 8.8 \\
\hline 128FGA-8 & 7.9 & 8.8 & 9.1 & 8.8 \\
\hline
\end{tabular}

*D1, D2, D3: Three independent diameter measurements of the same fiber at three different locations, see Methods for details. 
Table S18. Summary of mechanical properties of 128xFGAILSS hybrid proteins in this study.

\begin{tabular}{|l|l|l|l|l|l|}
\hline & $\begin{array}{l}\text { Ultimate } \\
\text { tensile stress } \\
\text { (MPa) }\end{array}$ & $\begin{array}{l}\text { Modulus } \\
(\mathbf{G P a})\end{array}$ & $\begin{array}{l}\text { Breaking } \\
\text { strain }(\mathbf{\%})\end{array}$ & $\begin{array}{l}\text { Toughness } \\
\left(\mathbf{M J}^{*-3}\right)\end{array}$ & $\begin{array}{l}\text { Diameter } \\
(\boldsymbol{\mu m})\end{array}$ \\
\hline 128FGA-1 & 1080 & 7.5 & 34 & 220 & 9.1 \\
\hline 128FGA-2 & 971 & 9.4 & 28 & 160 & 9.6 \\
\hline 128FGA-3 & 891 & 8.4 & 25 & 160 & 9.1 \\
\hline 128FGA-4 & 901 & 9.3 & 22 & 140 & 8.8 \\
\hline 128FGA-5 & 933 & 11.7 & 32 & 170 & 9.4 \\
\hline 128FGA-6 & 916 & 7.4 & 24 & 150 & 8.8 \\
\hline 128FGA-7 & 1074 & 12.7 & 22 & 150 & 8.3 \\
\hline 128FGA-8 & 1067 & 9.0 & 22 & 140 & 9.0 \\
\hline Average & 979 & 9.4 & 26 & 161 & 0.41 \\
\hline $\begin{array}{l}\text { Standard } \\
\text { Deviation }\end{array}$ & 82 & 1.9 & 4.6 & 26 & \\
\hline
\end{tabular}


Table S19. Fitted parameters from WAXD results.

\begin{tabular}{|c|c|c|c|c|c|}
\hline $\begin{array}{l}\text { Sample } \\
\text { Name }\end{array}$ & $\begin{array}{l}\text { Integration } \\
\text { Mode }\end{array}$ & Peak Index & $\begin{array}{l}\text { Peak } \\
\text { Center } \\
(\text { PC) }\end{array}$ & FWHM & Intensity (I) \\
\hline \multirow[t]{10}{*}{ KLVFFAE } & \multirow{5}{*}{$\begin{array}{l}\text { Radial } \\
\text { (Equatorial) }\end{array}$} & $(200)$ & $0.692\left(\AA^{-1}\right)$ & $0.620\left(\AA^{-1}\right)$ & 26.6 (a.u.) \\
\hline & & $(120)$ & $1.38\left(\AA^{-1}\right)$ & $0.143\left(\AA^{-1}\right)$ & 0.920 (a.u.) \\
\hline & & $\begin{array}{l}\text { Amorphous } \\
1\end{array}$ & $0.517\left(\AA^{-1}\right)$ & $0.361\left(\AA^{-1}\right)$ & 4.98 (a.u.) \\
\hline & & $\begin{array}{l}\text { Amorphous } \\
2\end{array}$ & $1.33\left(\AA^{-1}\right)$ & $1.21\left(\AA^{-1}\right)$ & 81.4 (a.u.) \\
\hline & & $\begin{array}{l}\text { Amorphous } \\
3\end{array}$ & $1.98\left(\AA^{-1}\right)$ & $2.29\left(\AA^{-1}\right)$ & 66.2 (a.u.) \\
\hline & \multirow{3}{*}{$\begin{array}{l}\text { Azimuthal } \\
\text { (120) }\end{array}$} & $(120)$ & $181 / 363\left(^{\circ}\right)$ & $28.3 / 29.1\left(^{\circ}\right)$ & 430/399 (a.u.) \\
\hline & & (201) & $\begin{array}{l}129 / 230 / \\
312 / 413\left(^{\circ}\right)\end{array}$ & $\begin{array}{l}26.6 / 49.9 / 13.3 / \\
16.1\left(^{\circ}\right)\end{array}$ & $\begin{array}{l}\text { 104/118/43.6/ } \\
53.8 \text { (a.u.) }\end{array}$ \\
\hline & & Amorphous & $181 / 363\left(^{\circ}\right)$ & $70.1 / 66.7\left(^{\circ}\right)$ & 400/626 (a.u.) \\
\hline & \multirow{2}{*}{$\begin{array}{l}\text { Azimuthal } \\
\text { (200) }\end{array}$} & $(200)$ & $183 / 359\left(^{\circ}\right)$ & $35.5 / 40.2\left(^{\circ}\right)$ & 217/308 (a.u.) \\
\hline & & Amorphous & $183 / 359\left(^{\circ}\right)$ & $80.8 / 78.3\left(^{\circ}\right)$ & 590/644 (a.u.) \\
\hline \multirow[t]{7}{*}{ GDVIEV } & \multirow{5}{*}{$\begin{array}{l}\text { Radial } \\
\text { (Equatorial) }\end{array}$} & $(200)$ & $0.692\left(\AA^{-1}\right)$ & $0.620\left(\AA^{-1}\right)$ & 35.0 (a.u.) \\
\hline & & $(120)$ & $1.38\left(\AA^{-1}\right)$ & $0.138\left(\AA^{-1}\right)$ & 1.67 (a.u.) \\
\hline & & $\begin{array}{l}\text { Amorphous } \\
1\end{array}$ & $0.495\left(\AA^{-1}\right)$ & $0.300\left(\AA^{-1}\right)$ & 3.80 (a.u.) \\
\hline & & $\begin{array}{l}\text { Amorphous } \\
2\end{array}$ & $1.38\left(\AA^{-1}\right)$ & $1.14\left(\AA^{-1}\right)$ & 82.3 (a.u.) \\
\hline & & $\begin{array}{l}\text { Amorphous } \\
3\end{array}$ & $1.97\left(\AA^{-1}\right)$ & $2.26\left(\AA^{-1}\right)$ & 78.7 (a.u.) \\
\hline & \multirow{2}{*}{$\begin{array}{l}\text { Azimuthal } \\
(120)\end{array}$} & $(120)$ & $177 / 359\left(^{\circ}\right)$ & $23.3 / 23.3\left(^{\circ}\right)$ & 494/407 (a.u.) \\
\hline & & $(201)$ & $\begin{array}{l}129 / 233 / \\
307 / 414\left(^{\circ}\right)\end{array}$ & $\begin{array}{l}23.0 / 34.5 / 16.3 / \\
27.2\left(^{\circ}\right)\end{array}$ & $\begin{array}{l}\text { 197/138/119/ } \\
145 \text { (a.u.) }\end{array}$ \\
\hline
\end{tabular}




\begin{tabular}{|c|c|c|c|c|c|}
\hline & & Amorphous & $177 / 359\left(^{\circ}\right)$ & $64.1 / 89.5\left(^{\circ}\right)$ & 329/744 (a.u.) \\
\hline & \multirow{2}{*}{$\begin{array}{l}\text { Azimuthal } \\
(200)\end{array}$} & $(200)$ & $178 / 358\left(^{\circ}\right)$ & $34.2 / 42.3\left(^{\circ}\right)$ & 266/389 (a.u.) \\
\hline & & Amorphous & $178 / 358\left(^{\circ}\right)$ & $96.2 / 88.1\left(^{\circ}\right)$ & 434/498 (a.u.) \\
\hline \multirow[t]{10}{*}{ FGAILSS } & \multirow{5}{*}{$\begin{array}{l}\text { Radial } \\
\text { (Equatorial) }\end{array}$} & $(200)$ & $0.693\left(\AA^{-1}\right)$ & $0.623\left(\AA^{-1}\right)$ & 36.0 (a.u.) \\
\hline & & $(120)$ & $1.39\left(\AA^{-1}\right)$ & $0.128\left(\AA^{-1}\right)$ & 1.45 (a.u.) \\
\hline & & $\begin{array}{l}\text { Amorphous } \\
1\end{array}$ & $0.473\left(\AA^{-1}\right)$ & $0.169\left(\AA^{-1}\right)$ & 0.981 (a.u.) \\
\hline & & $\begin{array}{l}\text { Amorphous } \\
2\end{array}$ & $1.41\left(\AA^{-1}\right)$ & $1.04\left(\AA^{-1}\right)$ & 63.9 (a.u.) \\
\hline & & $\begin{array}{l}\text { Amorphous } \\
3\end{array}$ & $1.78\left(\AA^{-1}\right)$ & $2.43\left(\AA^{-1}\right)$ & 93.6 (a.u.) \\
\hline & \multirow{3}{*}{$\begin{array}{l}\text { Azimuthal } \\
(120)\end{array}$} & $(120)$ & $180 / 360\left(^{\circ}\right)$ & $21.4 / 31.4\left(^{\circ}\right)$ & 455/645 (a.u.) \\
\hline & & (201) & $\begin{array}{l}128 / 232 / \\
307 / 415\left(^{\circ}\right)\end{array}$ & $\begin{array}{l}19.0 / 20.0 / 14.5 / \\
22.3\left(^{\circ}\right)\end{array}$ & $\begin{array}{l}\text { 80.0/142.4/98.9 } \\
/ 121 \text { (a.u.) }\end{array}$ \\
\hline & & Amorphous & $180 / 360\left(^{\circ}\right)$ & $63.7 / 70.0\left(^{\circ}\right)$ & 271/230 (a.u.) \\
\hline & \multirow{2}{*}{$\begin{array}{l}\text { Azimuthal } \\
(200)\end{array}$} & (200) & $179 / 359\left(^{\circ}\right)$ & $29.8 / 27.0\left(^{\circ}\right)$ & 330/315 (a.u.) \\
\hline & & Amorphous & $179 / 359\left(^{\circ}\right)$ & $75.2 / 74.8\left(^{\circ}\right)$ & $378 / 427$ (a.u.) \\
\hline \multirow{8}{*}{$\begin{array}{l}\text { Recombina } \\
\text { nt silk }\end{array}$} & \multirow{5}{*}{$\begin{array}{l}\text { Radial } \\
\text { (Equatorial) }\end{array}$} & $(200)$ & $1.10\left(\AA^{-1}\right)$ & $0.399\left(\AA^{-1}\right)$ & 3.19 (a.u.) \\
\hline & & $(120)$ & $1.41\left(\AA^{-1}\right)$ & $0.152\left(\AA^{-1}\right)$ & 1.26 (a.u.) \\
\hline & & $\begin{array}{l}\text { Amorphous } \\
1\end{array}$ & $0.635\left(\AA^{-1}\right)$ & $0.624\left(\AA^{-1}\right)$ & 18.9 (a.u.) \\
\hline & & $\begin{array}{l}\text { Amorphous } \\
2\end{array}$ & $1.41\left(\AA^{-1}\right)$ & $1.08\left(\AA^{-1}\right)$ & 37.9 (a.u.) \\
\hline & & $\begin{array}{l}\text { Amorphous } \\
3\end{array}$ & $1.93\left(\AA^{-1}\right)$ & $2.31\left(\AA^{-1}\right)$ & 44.6 (a.u.) \\
\hline & \multirow{3}{*}{$\begin{array}{l}\text { Azimuthal } \\
(120)\end{array}$} & $(120)$ & $179 / 358\left(^{\circ}\right)$ & $24.6 / 28.5\left(^{\circ}\right)$ & 389/442 (a.u.) \\
\hline & & (201) & $\begin{array}{l}126 / 235 / \\
306 / 416\left(^{\circ}\right)\end{array}$ & $\begin{array}{l}15.8 / 13.0 / 12.6 / \\
18.1\left(^{\circ}\right)\end{array}$ & $\begin{array}{l}70.0 / 63.9 / 81.4 / \\
153 \text { (a.u.) }\end{array}$ \\
\hline & & Amorphous & $179 / 358\left(^{\circ}\right)$ & $63.0 / 66.8\left(^{\circ}\right)$ & 417/391 (a.u.) \\
\hline
\end{tabular}




\begin{tabular}{|l|l|l|l|l|l|}
\hline \multirow{2}{*}{$\begin{array}{l}\text { Azimuthal } \\
(200)\end{array}$} & $(200)$ & $181 / 357\left(^{\circ}\right)$ & $29.4 / 33.2\left(^{\circ}\right)$ & $102 / 151$ (a.u.) \\
\cline { 3 - 6 } & Amorphous & $181 / 357\left(^{\circ}\right)$ & $75.1 / 61.6\left(^{\circ}\right)$ & $134 / 193$ (a.u.) \\
\hline
\end{tabular}


Table S20. Summary of mechanical properties of natural and proteinaceous silks reported in previous studies and this work.

\begin{tabular}{|c|c|c|c|c|}
\hline & $\begin{array}{l}\text { Ultimate tensile } \\
\text { stress (MPa) }\end{array}$ & $\begin{array}{l}\text { Toughness } \\
\left(\mathbf{M J} \mathbf{J}^{-3}\right)\end{array}$ & Diameter $(\boldsymbol{\mu m})$ & Reference \\
\hline $\begin{array}{l}\text { Natural } N . \quad \text { clavipes } \\
\text { dragline silk }\end{array}$ & $965 \pm 217$ & $111 \pm 30$ & $4.7 \pm 1.3$ & $\begin{array}{l}\text { Zemlin } \\
(1968),{ }^{1} \quad \text { Ko } \\
(2001){ }^{2} \\
\text { Swanson } \\
(2006)^{3}\end{array}$ \\
\hline $\begin{array}{l}\text { Natural A. trifasciata } \\
\text { dragline silk }\end{array}$ & $890 \pm 130$ & $100 \pm 40$ & $\sim 3$ & Plaza $(2012)^{4}$ \\
\hline $\begin{array}{l}\text { Natural } A . \quad \text { sericatus } \\
\text { dragline silk }\end{array}$ & 710 & 106 & N/A & Denny $(1976)^{5}$ \\
\hline $\begin{array}{l}\text { Natural } A . \text { trifasciata } \\
\text { minor ampullate silk }\end{array}$ & $483 \pm 34$ & $150 \pm 12$ & N/A & $\begin{array}{l}\text { Blackledge } \\
(2006)^{6}\end{array}$ \\
\hline Synthetic 96x & $508 \pm 108$ & N/A & N/A & $\mathrm{Xia}(2010)^{7}$ \\
\hline MaSp1/MaSp2 4:1 mix & $47 \pm 22$ & $42 \pm 8$ & N/A & An $(2012)^{8}$ \\
\hline 11RPC & $308 \pm 57$ & N/A & N/A & $\operatorname{Lin}(2013)^{9}$ \\
\hline NT2RepCT & $162 \pm 8$ & $45 \pm 7$ & $12 \pm 2$ & $\begin{array}{l}\text { Andersson } \\
(2017)^{10}\end{array}$ \\
\hline eADF3(AQ)12NR3 & $54 \pm 16$ & $2 \pm 0.8$ & $39 \pm 6$ & $\begin{array}{l}\text { Heidebrecht } \\
(2015)^{11}\end{array}$ \\
\hline SRT-ELP Cys36x & $603 \pm 18$ & $113 \pm 15$ & N/A & $\operatorname{Li}(2020)^{12}$ \\
\hline reSp2 & $330 \pm 20$ & $62 \pm 7$ & $36 \pm 2$ & Elices $(2011)^{13}$ \\
\hline Synthetic 192x & $1031 \pm 111$ & $114 \pm 51$ & $5.3 \pm 1.7$ & $\begin{array}{l}\text { Bowen } \\
(2018)^{14}\end{array}$ \\
\hline 128xFGAILSS & $979 \pm 82$ & $161 \pm 26$ & $9.0 \pm 0.4$ & This work \\
\hline
\end{tabular}




\section{Reference}

1. Zemlin, J. C., A Study of the Mechanical Behavior of Spider Silks. In Clothing and Organic Materials Laboratory Report, US Army Natick Labs: Natick, Massachusetts, 1968; pp 168.

2. Ko, F. K.; Kawabata, S.; Inoue, M.; Niwa, M.; Fossey, S.; Song, J. W., Engineering Properties of Spider Silk. MRS Proceedings 2001, 702.

3. Swanson, B. O.; Blackledge, T. A.; Summers, A. P.; Hayashi, C. Y., Spider Dragline Silk: Correlated and Mosaic Evolution in High-Performance Biological Materials. Evolution 2006, 60 (12), 2539-2551.

4. Plaza, G. R.; Pérez-Rigueiro, J.; Riekel, C.; Perea, G. B.; Agulló-Rueda, F.; Burghammer, M.; Guinea, G. V.; Elices, M., Relationship between Microstructure and Mechanical Properties in Spider Silk Fibers: Identification of Two Regimes in the Microstructural Changes. Soft Matter 2012, 8 (22).

5. Denny, M., Physical-Properties of Spiders Silk and Their Role in Design of Orb-Webs. $J$ Exp Biol 1976, 65 (2), 483-506.

6. Blackledge, T. A.; Hayashi, C. Y., Silken Toolkits: Biomechanics of Silk Fibers Spun by the Orb Web Spider Argiope Argentata (Fabricius 1775). J Exp Biol 2006, 209 (Pt 13), 2452-61.

7. Xia, X. X.; Qian, Z. G.; Ki, C. S.; Park, Y. H.; Kaplan, D. L.; Lee, S. Y., Native-Sized Recombinant Spider Silk Protein Produced in Metabolically Engineered Escherichia Coli Results in a Strong Fiber. Proc Natl Acad Sci U S A 2010, 107 (32), 14059-63. 
8. An, B.; Jenkins, J. E.; Sampath, S.; Holland, G. P.; Hinman, M.; Yarger, J. L.; Lewis, R., Reproducing Natural Spider Silks' Copolymer Behavior in Synthetic Silk Mimics. Biomacromolecules 2012, 13 (12), 3938-48.

9. Lin, Z.; Deng, Q.; Liu, X. Y.; Yang, D., Engineered Large Spider Eggcase Silk Protein for Strong Artificial Fibers. Adv Mater 2013, 25 (8), 1216-20.

10. Andersson, M.; Jia, Q.; Abella, A.; Lee, X. Y.; Landreh, M.; Purhonen, P.; Hebert, H.; Tenje, M.; Robinson, C. V.; Meng, Q.; Plaza, G. R.; Johansson, J.; Rising, A., Biomimetic Spinning of Artificial Spider Silk from a Chimeric Minispidroin. Nat Chem Biol 2017, 13 (3), 262264.

11. Heidebrecht, A.; Eisoldt, L.; Diehl, J.; Schmidt, A.; Geffers, M.; Lang, G.; Scheibel, T., Biomimetic Fibers Made of Recombinant Spidroins with the Same Toughness as Natural Spider Silk. Adv Mater 2015, 27 (13), 2189-94.

12. Li, Y.; Li, J.; Sun, J.; He, H.; Li, B.; Ma, C.; Liu, K.; Zhang, H., Bioinspired and Mechanically Strong Fibers Based on Engineered Non-Spider Chimeric Proteins. Angew Chem Int Ed Engl 2020, 59 (21), 8148-8152.

13. Elices, M.; Guinea, G. V.; Plaza, G. R.; Karatzas, C.; Riekel, C.; Agullo-Rueda, F.; Daza, R.; Perez-Rigueiro, J., Bioinspired Fibers Follow the Track of Natural Spider Silk. Macromolecules 2011, 44 (5), 1166-1176.

14. Bowen, C. H.; Dai, B.; Sargent, C. J.; Bai, W.; Ladiwala, P.; Feng, H.; Huang, W.; Kaplan, D. L.; Galazka, J. M.; Zhang, F., Recombinant Spidroins Fully Replicate Primary Mechanical Properties of Natural Spider Silk. Biomacromolecules 2018, 19 (9), 3853-3860. 
15. Swanson, B. O.; Blackledge, T. A.; Summers, A. P.; Hayashi, C. Y., Spider Dragline Silk: Correlated and Mosaic Evolution in High-Performance Biological Materials. Evolution 2006, 60 (12), 2539-51.

16. Yazawa, K.; Malay, A. D.; Masunaga, H.; Norma-Rashid, Y.; Numata, K., Simultaneous Effect of Strain Rate and Humidity on the Structure and Mechanical Behavior of Spider Silk. Commun Mater 2020, 1 (1). 